\title{
Dynamical analysis of a stochastic SIS epidemic model with nonlinear incidence rate and double epidemic hypothesis
}

\author{
Anqi Miao ${ }^{1}$, Xinyang Wang ${ }^{1}$, Tongqian Zhang ${ }^{1,2^{*}} \mathbb{D}$, Wei Wang ${ }^{3}$ and BG Sampath Aruna Pradeep ${ }^{4}$
}

"Correspondence:

zhangtongqian@sdust.edu.cn ${ }^{1}$ College of Mathematics and

Systems Science, Shandong

University of Science and

Technology, Qianwangang Road 579, Qingdao, 266590, P.R. China ${ }^{2}$ State Key Laboratory of Mining Disaster Prevention and Control Co-founded by Shandong Province and the Ministry of Science and Technology, Shandong University of Science and Technology, Qianwangang Road 579, Qingdao, 266590, P.R. China

Full list of author information is available at the end of the article

\begin{abstract}
In this paper, a stochastic SIS epidemic model with nonlinear incidence rate and double epidemic hypothesis is proposed and analysed. We explain the effects of stochastic disturbance on disease transmission. To this end, firstly, we investigated the dynamic properties of the system neglecting stochastic disturbance and obtained the threshold and the conditions for the extinction and the permanence of two kinds of epidemic diseases by considering the stability of the equilibria of the deterministic system. Secondly, we paid prime attention on the threshold dynamics of the stochastic system and established the conditions for the extinction and the permanence of two kinds of epidemic diseases. We found that there exists a significant difference between the threshold of the deterministic system and that of the stochastic system. Moreover, it has been established that the persistent of infectious disease analysed by use of deterministic system becomes extinct under the same conditions due to the stochastic disturbance. This implies that a stochastic disturbance has significant impact on the spread of infectious diseases and the larger stochastic disturbance leads to control the epidemic diseases. In order to illustrate the dynamic difference between the deterministic system and the stochastic system, there have been given a series of numerical simulations by using different noise disturbance coefficients.
\end{abstract}

MSC: $60 \mathrm{H} 10 ; 65 \mathrm{C} 30 ; 91 \mathrm{~B} 70$

Keywords: stochastic SIS epidemic model; double epidemic hypothesis; Beddington-DeAngelis incidence rate; extinction; permanence

\section{Springer}

\section{Introduction}

Infectious disease is generally considered as the enemy of human health; in history, the epidemic of infectious diseases such as smallpox, cholera, AIDS and so on have brought great disaster to the national economy of a country and people's livelihood [1]. In order to control the spread of infectious diseases, researchers have built a great deal of mathematical models to study the dynamical behavior of infectious diseases [2-6]. The mathematical models of differential equations play a significant role in describing the dynamic behavior and have been widely used in biology [7-11], physics [12-16], medicine [17, 18], and so on [19-24]. An important mathematical model describing the evolution infectious diseases is called 'compartmental model' which was originally established by Kermack and

(c) The Author(s) 2017. This article is distributed under the terms of the Creative Commons Attribution 4.0 International License (http://creativecommons.org/licenses/by/4.0/), which permits unrestricted use, distribution, and reproduction in any medium, provided you give appropriate credit to the original author(s) and the source, provide a link to the Creative Commons license, and indicate if changes were made. 
Mckendrick to study the spread of the infectious diseases such as Great Plague in London and the Plague of Mumbai [25]. In the compartmental system, the population is divided into three separate compartments, namely, the susceptible compartment $S$, the infected compartment $I$, and the removed compartment $R$. In the system, the susceptible person get infected and becomes an infected person making contact with an infected person, and the infected person can be recovered taking treatments, the individuals who reach this class have permanent immunity for the relevant disease. This type of model is called the SIR (susceptible-infected-removed) model, which can be mathematically expressed as (see p.47 in [25]):

$$
\left\{\begin{array}{l}
\dot{S}(t)=-\beta S(t) I(t), \\
\dot{I}(t)=\beta S(t) I(t)-\gamma I(t), \\
\dot{R}(t)=\gamma I(t),
\end{array}\right.
$$

where $S(t), I(t)$ and $R(t)$ represents the number of susceptible, infected and removed individuals at time $t$, respectively, $\beta$ is the contact rate, $\gamma$ is the recovery rate, $\beta S I$ is called bilinear infection rate. However, some diseases do not conform to the SIR system, such as influenza, infected individuals do not get permanent immunity for the disease although they take proper treatments, in this case, there exists a high possibility of recovered individuals to be re-infected. This type of model is called an SIS model, the mathematical system can be expressed in the following form (see p.62 in [26]):

$$
\left\{\begin{array}{l}
\dot{S}(t)=-d S(t)-\beta S(t) I(t)+\gamma I(t), \\
\dot{I}(t)=\beta S(t) I(t)-(d+\gamma) I(t),
\end{array}\right.
$$

where $d$ is the natural death rate and $\gamma$ is the recovery rate of the infective individuals. In systems (1) and (2) there has been used the bilinear infection rate, a common nonlinear infection rate. In the previous work, the researchers studied other types of nonlinear infection rates, for example, several epidemic models with saturated infection rates $\beta S I /(1+\alpha S)$ were discussed by Xu et al. [27, 28] and Zhang et al. [29]. A nonlinear incidence rate $\lambda S^{q} I^{p}$ was proposed by Liu et al. [30,31], while a nonlinear infection rate $\beta S I^{p} /\left(1+\alpha S^{q}\right)$ was considered by Hethcote et al. [32]. And a special case $p=q=2$ for $\beta S I^{p} /\left(1+\alpha S^{q}\right)$ was investigated by Ruan and Wang [33]. The nonlinear infection rate of the form Beddington-DeAngelis functional response $\beta S I /(1+a S+b I)$ was studied by Chen et al. [34]. For a more general nonlinear incidence rate, we refer the reader to Wang [35].

It is well known that random noise factors play an important role in the transmission of infectious diseases. Therefore, many scholars [36-43] have studied the impact of the stochastic epidemic system, various stochastic perturbation approaches have been introduced into epidemic models and have obtained excellent results. For example, the authors of [44-49] have considered a stochastic epidemic model with a Markov transform. A class of epidemic model which shows the effect of the random white noise has been studied by the researchers in the articles [50-64]. Further, in [65-70], the authors studied a class of stochastic epidemic models, in which the stochastic white noise is assumed to be proportional to $S, I$ and $R$. It can be seen following the literature that the authors of the articles [71, 72] analysed a stochastic epidemic model with two different kinds of perturbation. A stochastic epidemic model with Lévy jumps has been proposed and studied by 
the researchers [73-75]; the authors investigated stochastic perturbation around the positive equilibria of deterministic models (see, for example, [40, 41, 76-78]). Although there were limited numbers of publications in the recent literature considering time delay and stochastic behavior, the authors in [77] paid attention on the stochastic epidemic model with time delay.

Recently, Meng et al. [50] constructed a nonlinear stochastic SIS epidemic model with double epidemic hypothesis, in which the saturated incidence rates $\frac{\beta_{i} S I_{i}}{a_{i}+I_{1}}(i=1,2)$ is proposed. Then, based on the previous work, firstly, we propose a deterministic epidemic model with Beddington-DeAngelis nonlinear incidence rate and double epidemic hypothesis as follows:

$$
\left\{\begin{aligned}
\dot{S}(t)= & A-d S(t)-\frac{\beta_{1} S(t) I_{1}(t)}{1+a_{1} S(t)+b_{1} I_{1}(t)}-\frac{\beta_{2} S(t) I_{2}(t)}{1+a_{2} S(t)+b_{2} I_{2}(t)} \\
& +r_{1} I_{1}(t)+r_{2} I_{2}(t) \\
\dot{I}_{1}(t)= & \frac{\beta_{1} S(t) I_{1}(t)}{1+a_{1} S(t)+b_{1} I_{1}(t)}-\left(d+\alpha_{1}+r_{1}\right) I_{1}(t) \\
\dot{I}_{2}(t)= & \frac{\beta_{2} S(t) I_{2}(t)}{1+a_{2} S(t)+b_{2} I_{2}(t)}-\left(d+\alpha_{2}+r_{2}\right) I_{2}(t),
\end{aligned}\right.
$$

where $S(t)$ denotes the number of the population susceptible to the disease, $I_{1}(t)$ and $I_{2}(t)$ are the total population of the infectives with virus $V_{A}$ and $V_{B}$ at time $t$, respectively. The recruitment to the susceptible population is to be considered as a constant $A, \beta_{1}$ and $\beta_{2}$ are the contact rates, $d$ is natural mortality rate, $\alpha_{1}$ and $\alpha_{2}$ are the rates of disease-related death, $r_{1}$ and $r_{2}$ are the treatment cure rates of two diseases, respectively. $a_{i}, b_{i}$ are the parameters that measure the inhibitory effect. The infection rate $\frac{\beta_{i} S(t) I_{i}(t)}{1+a_{i} S(t)+b_{i} I_{i}(t)}(i=1,2)$ of susceptible individuals through their contacts with infectious, includes three forms: The first one is the bilinear incidence rate $\beta S(t) I_{i}(t)$ for the case $a_{i}=b_{i}=0$; the second one is the saturated incidence rate for the susceptible with the form $\frac{\beta S(t) I_{i}(t)}{1+a_{i} S(t)}$ for the case $a_{i}>0, b_{i}=0$; and the third one is the saturated incidence rate for the infectives with the form $\frac{\beta S(t) I_{i}(t)}{1+b_{i} I(t)}$ for the case $a_{i}=0, b_{i}>0$. Thus, the nonlinear incidence rates $\frac{\beta_{i} S(t) I_{i}(t)}{1+a_{i} S(t)+b_{i} I_{i}(t)}(i=1,2)$ are more general and realistic than the saturated incidence rate $\frac{\beta S(t) I_{i}(t)}{1+a_{i} S(t)}$ and $\frac{\beta S(t) I_{i}(t)}{1+b_{i} I(t)}$, because it takes into account the inhibition effect of the susceptible and the infectives.

Secondly, we assume that fluctuations in the environment will manifest themselves mainly as fluctuations in the saturated response rate

$$
\frac{\beta_{i} S(t) I_{i}(t)}{1+a_{i} S(t)+b_{i} I_{i}(t)} \rightarrow \frac{\beta_{i} S(t) I_{i}(t)}{1+a_{i} S(t)+b_{i} I_{i}(t)}+\frac{\sigma_{i} S(t) I_{i}(t)}{1+a_{i} S(t)+b_{i} I_{i}(t)} \mathrm{d} B_{i}(t)
$$

where $B(t)=\left(B_{1}(t), B_{2}(t)\right)$ is the standard Brownian motion with intensity $\sigma_{i}>0(i=1,2)$. Finally, a stochastic version of system (3) is obtained as follows:

$$
\left\{\begin{aligned}
\mathrm{d} S(t)= & \left(A-d S(t)-\frac{\beta_{1} S(t) I_{1}(t)}{1+a_{1} S(t)+b_{1} I_{1}(t)}-\frac{\beta_{2} S(t) I_{2}(t)}{1+a_{2} S(t)+b_{2} I_{2}(t)}\right. \\
& \left.+r_{1} I_{1}(t)+r_{2} I_{2}(t)\right) \mathrm{d} t-\frac{\sigma_{1} S(t) I_{1}(t)}{1+a_{1} S(t)+b_{1} I_{1}(t)} \mathrm{d} B_{1}(t) \\
& -\frac{\sigma_{2} S(t) I_{2}(t)}{1+a_{2} S(t)+b_{2} I_{2}(t)} \mathrm{d} B_{2}(t), \\
\mathrm{d} I_{1}(t)= & \left(\frac{\beta_{1} S(t) I_{1}(t)}{1+a_{1} S(t)+b_{1} I_{1}(t)}-\left(d+\alpha_{1}+r_{1}\right) I_{1}(t)\right) \mathrm{d} t \\
& +\frac{\sigma_{1} S(t) I_{1}(t)}{1+a_{1} S(t)+b_{1} I_{1}(t)} \mathrm{d} B_{1}(t), \\
\mathrm{d} I_{2}(t)= & \left(\frac{\beta_{2} S(t) I_{2}(t)}{1+a_{2} S(t)+b_{2} I_{2}(t)}-\left(d+\alpha_{2}+r_{2}\right) I_{2}(t)\right) \mathrm{d} t \\
& +\frac{\sigma_{2} S(t) I_{2}(t)}{1+a_{2} S(t)+b_{2} I_{2}(t)} \mathrm{d} B_{2}(t),
\end{aligned}\right.
$$


where the parameters of system (4) have the same biological meaning as in system (3). The rest of the paper, we will dedicate to study the deterministic system (3) and the stochastic system (4) with nonlinear incidence rate and double epidemic hypothesis. The main aims of this paper are (a) to establish a set of most suitable conditions such that diseases to be died out or to be persistent, and (b) to obtain the thresholds (based on the basic reproductive number) of the above two SIS epidemic models.

\section{Preliminaries and lemmas}

In this section, we will give some notations, definitions and some lemmas which will be used for analysing our main results.

Throughout this paper, let $\left(\Omega, \mathcal{F},\{\mathcal{F}\}_{t \geq 0}, \mathcal{P}\right)$ be a complete probability space with a filtration $\left\{\mathcal{F}_{t}\right\}_{t \geq 0}$ satisfying the usual conditions (i.e. it is increasing and right continuous while $\mathcal{F}_{0}$ contains all $\mathcal{P}$-null sets $\left.R_{+}^{3}=\left\{x_{i}>0, i=1,2,3\right\}\right)$. The function $B(t)$ denotes a scalar Brownian motion defined on the complete probability space $\Omega$. For an integrable function $f$ on $[0,+\infty)$, define $\langle f(t)\rangle=\frac{1}{t} \int_{0}^{t} f(\theta) \mathrm{d} \theta$.

Definition $2.1([50,68])$

(i) The diseases $I_{1}(t)$ and $I_{2}(t)$ are said to be extinctive if $\lim _{t \rightarrow+\infty} I_{1}(t)=0$ and $\lim _{t \rightarrow+\infty} I_{2}(t)=0$.

(ii) The diseases $I_{1}(t)$ and $I_{2}(t)$ are said to be permanent in mean if there exist two positive constants $\lambda_{1}$ and $\lambda_{2}$ such that $\liminf _{t \rightarrow+\infty}\left\langle I_{1}(t)\right\rangle \geq \lambda_{1}$ and $\liminf _{t \rightarrow+\infty}\left\langle I_{2}(t)\right\rangle \geq \lambda_{2}$.

Lemma 2.1 For any initial value $\left(S(0), I_{1}(0), I_{2}(0)\right) \in R_{+}^{3}$, there exists a unique solution $\left(S(t), I_{1}(t), I_{2}(t)\right)$ to system (4) on $t \geq 0$, and the solution will remain in $R_{+}^{3}$ with probability 1 , namely, $\left(S(t), I_{1}(t), I_{2}(t)\right) \in R_{+}^{3}$ for all $t \geq 0$ almost surely.

Proof Since the coefficients of system (4) are locally Lipschitz continuous for any given initial value $\left(S(0), I_{1}(0), I_{2}(0)\right) \in R_{+}^{3}$, then by the work of Mao et al. [36], there is a unique local solution $\left(S(t), I_{1}(t), I_{2}(t)\right)$ on $t \in[0, \tau)$, where $\tau$ is the explosion time (see [58]). To show that this solution is global, we need to show that $\tau_{\infty}=\infty$ almost surely. To do so, let $\varepsilon_{0} \geq 0$ such that $S(0)>\varepsilon_{0}, I_{1}(0)>\varepsilon_{0}, I_{2}(0)>\varepsilon_{0}$. For any positive $\varepsilon \leq \varepsilon_{0}$, define the stopping time as follows:

$$
\tau_{\varepsilon}=\inf \left\{t \in\left[0, \tau_{e}\right): S(t) \leq \varepsilon \text { or } I_{1}(t) \leq \varepsilon \text { or } I_{2}(t) \leq \varepsilon\right\}
$$

where throughout this paper, we set inf $\varnothing=\infty$ (in the usual notation, $\varnothing$ denotes the empty set). Clearly, $\tau_{\varepsilon}$ is increasing as $\varepsilon \rightarrow 0$. Set $\tau_{0}=\lim _{\varepsilon \rightarrow 0} \tau_{\varepsilon}$, whence $\tau_{0} \leq \tau_{\varepsilon}$ almost surely. If we can show $\tau_{0}=\infty$ almost surely, then $\tau_{e}=\infty$ and $\left(S(t), I_{1}(t), I_{2}(t)\right) \in R_{+}^{3}$ for all $t \geq 0$ almost surely. In other words, to complete the proof we only need to show that $\tau_{0}=\infty$ almost surely.

If this statement is false, then there is a pair of constants $T>0$ and $\delta \in(0,1)$ such that $P\left\{\tau_{0} \leq T\right\}>\delta$. Hence, there is a positive constant $\varepsilon_{1} \leq \varepsilon_{0}$ such that $P\left\{\tau_{\varepsilon} \leq T\right\}$ for any positive $\varepsilon \leq \varepsilon_{1}$.

Besides, for $t<\tau_{e}$, we see that

$$
\begin{aligned}
d\left(S(t)+I_{1}(t)+I_{2}(t)\right) & =\left[A-d\left(S(t)+I_{1}(t)+I_{2}(t)\right)-\alpha_{1} I_{1}(t)-\alpha_{2} I_{2}(t)\right] \mathrm{d} t \\
& \leq\left[A-d\left(S(t)+I_{1}(t)+I_{2}(t)\right)\right] \mathrm{d} t
\end{aligned}
$$


and

$$
S(t)+I_{1}(t)+I_{2}(t) \leq \max \left\{S(0)+I_{1}(0)+I_{2}(0), \frac{A}{d}\right\}:=C_{1} .
$$

Define a function

$$
V\left(S(t), I_{1}(t), I_{2}(t)\right)=-\ln \frac{S(t)}{C_{1}}-\ln \frac{I_{1}(t)}{C_{1}}-\ln \frac{I_{2}(t)}{C_{1}} .
$$

Obviously, $V$ is positive definite. Using the Itô formula, we get

$$
\mathrm{d} V=L V \mathrm{~d} t+\frac{\sigma_{1}\left(I_{1}(t)-S(t)\right)}{1+a_{1} S(t)+b_{1} I_{1}(t)} \mathrm{d} B_{1}(t)+\frac{\sigma_{2}\left(I_{2}(t)-S(t)\right)}{1+a_{2} S(t)+b_{2} I_{2}(t)} \mathrm{d} B_{2}(t)
$$

where

$$
\begin{aligned}
L V= & -\frac{A+r_{1} I_{1}(t)+r_{2} I_{2}(t)}{S(t)}+3 d+\alpha_{1}+r_{1}+\alpha_{2}+r_{2} \\
& +\frac{\beta_{1}\left(I_{1}(t)-S(t)\right)}{1+a_{1} S(t)+b_{1} I_{1}(t)}+\frac{\beta_{2}\left(I_{2}(t)-S(t)\right)}{1+a_{2} S(t)+b_{2} I_{2}(t)} \\
& +\frac{\sigma_{1}^{2}\left(I_{1}(t)^{2}+S(t)^{2}\right)}{2\left(1+a_{1} S(t)+b_{1} I_{1}(t)\right)^{2}}+\frac{\sigma_{2}^{2}\left(I_{2}(t)^{2}+S(t)^{2}\right)}{2\left(1+a_{2} S(t)+b_{2} I_{2}(t)\right)^{2}} .
\end{aligned}
$$

By using (6), we can obtain

$$
\begin{aligned}
L V \leq & 3 d+\alpha_{1}+r_{1}+\alpha_{2}+r_{2}+\frac{\beta_{1} I_{1}(t)}{1+a_{1} S(t)+b_{1} I_{1}(t)}+\frac{\beta_{2} I_{2}(t)}{1+a_{2} S(t)+b_{2} I_{2}(t)} \\
& +\frac{\sigma_{1}^{2}\left(I_{1}(t)^{2}+S(t)^{2}\right)}{2\left(1+a_{1} S(t)+b_{1} I_{1}(t)\right)^{2}}+\frac{\sigma_{2}^{2}\left(I_{2}(t)^{2}+S(t)^{2}\right)}{2\left(1+a_{2} S(t)+b_{2} I_{2}(t)\right)^{2}} \\
\leq & 3 d+\alpha_{1}+r_{1}+\alpha_{2}+r_{2}+\beta_{1} C_{1}+\beta_{2} C_{1}+\sigma_{1}^{2} C_{1}^{2}+\sigma_{2}^{2} C_{1}^{2}:=C_{2} .
\end{aligned}
$$

Therefore,

$$
\mathrm{d} V \leq C_{2} \mathrm{~d} t+\frac{\sigma_{1}\left(I_{1}(t)-S(t)\right)}{1+a_{1} S(t)+b_{1} I_{1}(t)} \mathrm{d} B_{1}(t)+\frac{\sigma_{2}\left(I_{2}(t)-S(t)\right)}{1+a_{2} S(t)+b_{2} I_{2}(t)} \mathrm{d} B_{2}(t)
$$

Integrating both sides from 0 to $\tau_{\varepsilon} \wedge T$, by considering expectations, yields

$$
E V\left(S\left(\tau_{\varepsilon} \wedge T\right), I_{1}\left(\tau_{\varepsilon} \wedge T\right), I_{2}\left(\tau_{\varepsilon} \wedge T\right)\right) \leq V\left(S(0), I_{1}(0), I_{2}(0)\right)+C_{2} T .
$$

Set $\Omega_{\varepsilon}=\left\{\tau_{\varepsilon} \leq T\right\}$ for any positive $\varepsilon \leq \varepsilon_{1}$ and then $P\left(\Omega_{\varepsilon}>\delta\right)$. Note that, for every $\omega \in \Omega_{\varepsilon}$, there is at least one of $S\left(\tau_{\varepsilon}, \omega\right), I_{1}\left(\tau_{\varepsilon}, \omega\right), I_{2}\left(\tau_{\varepsilon}, \omega\right)$ equal to $\varepsilon$, then

$$
V\left(S\left(\tau_{\varepsilon}\right), I_{1}\left(\tau_{\varepsilon}\right), I_{2}\left(\tau_{\varepsilon}\right)\right) \geq-\ln \frac{\varepsilon}{C_{1}}
$$


Consequently,

$$
\begin{aligned}
V\left(S(0), I_{1}(0), I_{2}(0)\right)+C_{2} T & \geq E\left[I_{\Omega_{\varepsilon}} V\left(S\left(\tau_{\varepsilon} \wedge T\right), I_{1}\left(\tau_{\varepsilon} \wedge T\right), I_{2}\left(\tau_{\varepsilon} \wedge T\right)\right)\right] \\
& =P\left(\Omega_{\varepsilon}\right) V\left(S\left(\tau_{\varepsilon}\right), I_{1}\left(\tau_{\varepsilon}\right), I_{2}\left(\tau_{\varepsilon}\right)\right) \\
& >-\delta \ln \frac{\varepsilon}{C_{1}}
\end{aligned}
$$

where $I_{\Omega_{\varepsilon}}$ is the indicator function of $\Omega_{\varepsilon}$. Letting $\varepsilon \rightarrow 0$ leads to the contradiction

$$
\infty>V\left(S(0), I_{1}(0), I_{2}(0)\right)+C_{2} T=\infty
$$

Therefore, we must have $\tau_{0}=\infty$ almost surely. The proof of Lemma 2.1 is completed.

Lemma 2.2 Denote $\Gamma=\left\{\left(S(t), I_{1}(t), I_{2}(t)\right) \in R_{+}^{3}: S(t), I_{1}(t), I_{2}(t) \leq \frac{A}{d}, t \geq 0\right\}$, then $\Gamma$ is an invariant set on system (3) or (4).

Proof From system (3) or the system (4), we have

$$
\frac{\mathrm{d}\left(S(t)+I_{1}(t)+I_{2}(t)\right)}{\mathrm{d} t} \leq A-d\left(S(t)+I_{1}(t)+I_{2}(t)\right) .
$$

This implies that

$$
S(t)+I_{1}(t)+I_{2}(t) \leq \frac{A}{d}+\left(S(0)+I_{1}(0)+I_{2}(0)-\frac{A}{d}\right) e^{-d t} .
$$

Then, if we denote $\Gamma=\left\{\left(S(t), I_{1}(t), I_{2}(t)\right) \in R_{+}^{3}: S(t), I_{1}(t), I_{2}(t) \leq \frac{A}{d}, t \geq 0\right\}$, we have $S(t)+$ $I_{1}(t)+I_{2}(t) \leq \frac{A}{d}$. Thus, the region $\Gamma$ is positively invariant.

By Lemma 2.2 and the strong law of large numbers for martingales [36], we can obtain the following lemma.

Lemma 2.3 Let $\left(S(t), I_{1}(t), I_{2}(t)\right)$ be a solution of system (4) with initial value $\left(S(0), I_{1}(0)\right.$, $\left.I_{2}(0)\right) \in R_{+}^{3}$. Then

$$
\lim _{t \rightarrow+\infty} \frac{1}{t} \int_{0}^{t} \frac{\sigma_{i} S(\tau)}{1+a_{i} S(\tau)+b_{i} I_{i}(\tau)} \mathrm{d} B_{i}(\tau)=0, \quad \lim _{t \rightarrow+\infty} \frac{1}{t} \int_{0}^{t} \sigma_{i} S(\tau) \mathrm{d} B_{i}(\tau)=0, \quad i=1,2 .
$$

\section{Dynamics of deterministic system (3)}

In this section, we will qualitatively analyse the dynamics of deterministic system (3). Firstly, to find the equilibria of system (3), we consider the following set of equations:

$$
\left\{\begin{aligned}
f_{1}:= & A-d S(t)-\frac{\beta_{1} S(t) I_{1}(t)}{1+a_{1} S(t)+b_{1} I_{1}(t)}-\frac{\beta_{2} S(t) I_{2}(t)}{1+a_{2} S(t)+b_{2} I_{2}(t)} \\
& +r_{1} I_{1}(t)+r_{2} I_{2}(t)=0 \\
f_{2}:= & \frac{\beta_{1} S(t) I_{1}(t)}{1+a_{1} S(t)+b_{1} I_{1}(t)}-\left(d+\alpha_{1}+r_{1}\right) I_{1}(t)=0 \\
f_{3}:= & \frac{\beta_{2} S(t) I_{2}(t)}{1+a_{2} S(t)+b_{2} I_{2}(t)}-\left(d+\alpha_{2}+r_{2}\right) I_{2}(t)=0 .
\end{aligned}\right.
$$


By direct calculation, there can be obtained the following equilibria for system (3):

$$
\begin{aligned}
E_{0}: & \left(\frac{A}{d}, 0,0\right), \\
E_{1}: \quad & \left(S_{1}^{*}, I_{1}^{*}, 0\right) \quad \text { with } \\
& S_{1}^{*}=\frac{\left(d+\alpha_{1}+r_{1}\right)\left(1+b_{1} I_{1}^{*}\right)}{\beta_{1}-\left(d+\alpha_{1}+r_{1}\right) a_{1}}, \\
& I_{1}^{*}=\frac{A \beta_{1}-\left(A a_{1}+d\right)\left(d+\alpha_{1}+r_{1}\right)}{d\left(d+\alpha_{1}+r_{1}\right) b_{1}+\left(d+\alpha_{1}\right)\left(\beta_{1}-\left(d+\alpha_{1}+r_{1}\right) a_{1}\right)},
\end{aligned}
$$

$E_{2}: \quad\left(S_{2}^{*}, 0, I_{2}^{*}\right) \quad$ with

$$
\begin{aligned}
& S_{2}^{*}=\frac{\left(d+\alpha_{2}+r_{2}\right)\left(1+b_{2} I_{2}^{*}\right)}{\beta_{2}-\left(d+\alpha_{2}+r_{2}\right) a_{2}}, \\
& I_{2}^{*}=\frac{A \beta_{2}-\left(A a_{2}+d\right)\left(d+\alpha_{2}+r_{2}\right)}{d\left(d+\alpha_{2}+r_{2}\right) b_{2}+\left(d+\alpha_{2}\right)\left(\beta_{2}-\left(d+\alpha_{2}+r_{2}\right) a_{2}\right)},
\end{aligned}
$$

$E^{*}: \quad\left(S^{*}, I_{1}^{* *}, I_{2}^{* *}\right) \quad$ with

$$
\begin{aligned}
S^{*} & =\frac{\left(A b_{1} b_{2}+C_{1} b_{2}+C_{2} b_{1}\right) B_{1} B_{2}}{d b_{1} b_{2} B_{1} B_{2}+C_{1} b_{2} B_{2}\left(\beta_{1}-B_{1} a_{1}\right)+C_{2} b_{1} B_{1}\left(\beta_{2}-B_{2} a_{2}\right)}, \\
I_{1}^{* *} & =\frac{S^{*}\left(\beta_{1}-B_{1} a_{1}\right)-B_{1}}{B_{1} b_{1}}, \\
I_{2}^{* *} & =\frac{S^{*}\left(\beta_{2}-B_{2} a_{2}\right)-B_{2}}{B_{2} b_{2}},
\end{aligned}
$$

where

$$
B_{1}=d+\alpha_{1}+r_{1}, \quad B_{2}=d+\alpha_{2}+r_{2}, \quad C_{1}=d+\alpha_{1}, \quad C_{2}=d+\alpha_{2} .
$$

For convenience, let us denote following expressions as $Q_{1}, Q_{2}$ and $Q_{3}$ and the combination of these three conditions are denoted by a single character $H$,

$$
\begin{aligned}
& Q_{1}=\left(A b_{2} a_{1}+d b_{2}+C_{2} a_{1}\right)\left(R_{1}-1\right)-C_{2} a_{2}\left(R_{2}-1\right)+C_{2} \frac{d}{A}\left(R_{1}-R_{2}\right)>0, \\
& Q_{2}=\left(A b_{1} a_{2}+d b_{1}+C_{1} a_{2}\right)\left(R_{2}-1\right)-C_{1} a_{1}\left(R_{1}-1\right)+C_{1} \frac{d}{A}\left(R_{2}-R_{1}\right)>0, \\
& Q_{3}=\left(d b_{1} b_{2} B_{1} B_{2}+C_{1} b_{2} B_{2}\left(\beta_{1}-B_{1} a_{1}\right)+C_{2} b_{1} B_{1}\left(\beta_{2}-B_{2} a_{2}\right)\right)>0 .
\end{aligned}
$$

Therefore, there exist a unique positive equilibrium $E^{*}$ for system (3) if $H$ holds. Thus, if let

$$
\mathcal{R}_{1}=\frac{\beta_{1} A}{\left(A a_{1}+d\right)\left(d+\alpha_{1}+r_{1}\right)}, \quad \mathcal{R}_{2}=\frac{\beta_{2} A}{\left(A a_{2}+d\right)\left(d+\alpha_{2}+r_{2}\right)},
$$

then we have the following theorem.

Theorem 3.1 For system (3), the following conclusions are true:

(i) if $\mathcal{R}_{1}<1$ and $\mathcal{R}_{2}<1$, then both diseases go extinct and system (3) has a unique stable 'diseases-extinction' equilibrium $E_{0}$; 
(ii) if $\mathcal{R}_{1}>1$ and $\mathcal{R}_{2}<1$, then the disease $I_{2}$ goes extinct and system (3) has a unique stable equilibrium $E_{1}$;

(iii) if $\mathcal{R}_{1}<1$ and $\mathcal{R}_{2}>1$, then the disease $I_{1}$ goes extinct and system (3) has a unique stable equilibrium $E_{2}$; and

(iv) when condition $H$ holds, and if $\mathcal{R}_{1}>1$ and $\mathcal{R}_{2}>1$, then $E^{*}$ is a unique stable equilibrium, which implies both diseases of system (3) are permanent.

Proof Let $S, I_{1}, I_{2}$ be an arbitrary equilibrium of system (3), then the Jacobian matrix associating to the corresponding equilibrium of system (3) is

$$
J=\left(\begin{array}{lll}
a_{11} & a_{12} & a_{13} \\
a_{21} & a_{22} & a_{23} \\
a_{31} & a_{32} & a_{33}
\end{array}\right)
$$

Here

$$
\begin{aligned}
& a_{11}=-d-\frac{\beta_{1}\left(I_{1}+b_{1} I_{1}^{2}\right)}{\left(1+a_{1} S+b_{1} I_{1}\right)^{2}}-\frac{\beta_{2}\left(I_{2}+b_{2} I_{2}^{2}\right)}{\left(1+a_{2} S+b_{2} I_{2}\right)^{2}}, \\
& a_{12}=r_{1}-\frac{\beta_{1}\left(S+a_{1} S^{2}\right)}{\left(1+a_{1} S+b_{1} I_{1}\right)^{2}}, \\
& a_{13}=r_{2}-\frac{\beta_{2}\left(S+a_{2} S^{2}\right)}{\left(1+a_{2} S+b_{2} I_{2}\right)^{2}}, \\
& a_{21}=\frac{\beta_{1}\left(I_{1}+b_{1} I_{1}^{2}\right)}{\left(1+a_{1} S+b_{1} I_{1}\right)^{2}}, \\
& a_{22}=\frac{\beta_{1}\left(S+a_{1} S^{2}\right)}{\left(1+a_{1} S+b_{1} I_{1}\right)^{2}}-\left(d+\alpha_{1}+r_{1}\right), \\
& a_{23}=0, \\
& a_{31}=\frac{\beta_{2}\left(I_{2}+b_{2} I_{2}^{2}\right)}{\left(1+a_{2} S+b_{2} I_{2}\right)^{2}}, \\
& a_{32}=0, \\
& a_{33}=\frac{\beta_{2}\left(S+a_{2} S^{2}\right)}{\left(1+a_{2} S+b_{2} I_{2}\right)^{2}}-\left(d+\alpha_{2}+r_{2}\right) .
\end{aligned}
$$

The stability of the 'diseases-extinction' equilibrium $\left(\frac{A}{d}, 0,0\right)$ of system $(3)$ is determined by the Jacobian matrix

$$
J_{0}=\left(\begin{array}{ccc}
-d & r_{1}-\frac{\beta_{1} A}{d} & r_{2}-\frac{\beta_{2} A}{d} \\
0 & \frac{\beta_{1} S}{1+a_{1} S}-\left(d+\alpha_{1}+r_{1}\right) & 0 \\
0 & 0 & \frac{\beta_{2} S}{1+a_{2} S}-\left(d+\alpha_{2}+r_{2}\right)
\end{array}\right)
$$

which has following eigenvalues:

$$
\lambda_{1}=-d<0, \quad \lambda_{2}=\frac{\beta_{1} A}{A a_{1}+d}-\left(d+\alpha_{1}+r_{1}\right), \quad \lambda_{3}=\frac{\beta_{2} A}{A a_{2}+d}-\left(d+\alpha_{2}+r_{2}\right) .
$$

According to stability theory, $\left(\frac{A}{d}, 0,0\right)$ is stable if $\lambda_{2}<0$ and $\lambda_{3}<0$, i.e., $\mathcal{R}_{1}<1$ and $\mathcal{R}_{2}<1$. 
At equilibrium $E_{1}$, the Jacobian matrix can be expressed as

$$
J_{1}=\left(\begin{array}{lll}
a_{11}^{*} & a_{12}^{*} & a_{13}^{*} \\
a_{21}^{*} & a_{22}^{*} & a_{23}^{*} \\
a_{31}^{*} & a_{32}^{*} & a_{33}^{*}
\end{array}\right),
$$

where

$$
\begin{aligned}
& a_{11}^{*}=-d-\frac{\beta_{1}\left(I_{1}^{*}+b_{1} I_{1}^{* 2}\right)}{\left(1+a_{1} S_{1}^{*}+b_{1} I_{1}^{*}\right)^{2}}, \\
& a_{12}^{*}=r_{1}-\frac{\beta_{1}\left(S_{1}^{*}+a_{1} S_{1}^{* 2}\right)}{\left(1+a_{1} S_{1}^{*}+b_{1} I_{1}^{*}\right)^{2}}, \\
& a_{13}^{*}=r_{2}-\frac{\beta_{2} S_{1}^{*}}{\left(1+a_{2} S_{1}^{*}\right)}, \\
& a_{21}^{*}=-\frac{\beta_{1}\left(I_{1}^{*}+b_{1} I_{1}^{* 2}\right)}{\left(1+a_{1} S_{1}^{*}+b_{1} I_{1}^{*}\right)^{2}}, \\
& a_{22}^{*}=\frac{\beta_{1}\left(S_{1}^{*}+a_{1} S_{1}^{* 2}\right)}{\left(1+a_{1} S_{1}^{*}+b_{1} I_{1}^{*}\right)^{2}}-\left(d+\alpha_{1}+r_{1}\right), \\
& a_{23}^{*}=0, \\
& a_{31}^{*}=0, \\
& a_{32}^{*}=0, \\
& a_{33}^{*}=\frac{\beta_{2} S_{1}^{*}}{1+a_{2} S_{1}^{*}}-\left(d+\alpha_{2}+r_{2}\right),
\end{aligned}
$$

and one of three eigenvalues of matrix $J_{1}$ is given by

$$
\lambda_{1}=a_{33}^{*}=\frac{\beta_{2} S_{1}^{*}}{1+a_{2} S_{1}^{*}}-\left(d+\alpha_{2}+r_{2}\right)<\frac{\left(\frac{d}{A} S_{1}^{*}-1\right)\left(d+\alpha_{2}+r_{2}\right)}{1+a_{2} S_{1}^{*}}<0,
$$

where $A-d S_{1}^{*}=\left(d+\alpha_{1}\right) I_{1}^{*}>0$ is used. The other two eigenvalues $\lambda_{2}$ and $\lambda_{3}$ of matrix $J_{1}$ are the roots of the following equation:

$$
\lambda^{2}+\left(a_{11}^{*}+a_{22}^{*}\right) \lambda+a_{11}^{*} a_{22}^{*}-a_{21}^{*} a_{12}^{*}=0
$$

Obviously, $a_{11}^{*}+a_{22}^{*}=\frac{\beta_{1}\left(I_{1}^{*}+b_{1} I_{1}^{* 2}\right)}{\left(1+a_{1} S_{1}^{*}+b_{1} I_{1}^{*}\right)^{2}}+d+\frac{\beta_{1} b_{1} S_{1}^{*} I_{1}^{*}}{\left(1+a_{1} S_{1}^{*}+b_{1} I_{1}^{*}\right)^{2}}>0$ and

$$
\begin{aligned}
a_{11}^{*} & a_{22}^{*}-a_{21}^{*} a_{12}^{*} \\
= & \left(\frac{\beta_{1}\left(I_{1}^{*}+b_{1} I_{1}^{* 2}\right)}{\left(1+a_{1} S_{1}^{*}+b_{1} I_{1}^{*}\right)^{2}}+d\right)\left(\left(d+\alpha_{1}+r_{1}\right)-\frac{\beta_{1}\left(S_{1}^{*}+a_{1} S_{1}^{* 2}\right)}{\left(1+a_{1} S_{1}^{*}+b_{1} I_{1}^{*}\right)^{2}}\right) \\
& \quad-\frac{\beta_{1}\left(I_{1}^{*}+b_{1} I_{1}^{* 2}\right)}{\left(1+a_{1} S_{1}^{*}+b_{1} I_{1}^{*}\right)^{2}}\left(r_{1}-\frac{\beta_{1}\left(S_{1}^{*}+a_{1} S_{1}^{* 2}\right)}{\left(1+a_{1} S_{1}^{*}+b_{1} I_{1}^{*}\right)^{2}}\right) \\
= & \left(d+\alpha_{1}\right) \frac{\beta_{1}\left(I_{1}^{*}+b_{1} I_{1}^{* 2}\right)}{\left(1+a_{1} S_{1}^{*}+b_{1} I_{1}^{*}\right)^{2}}+d\left(d+\alpha_{1}+r_{1}\right)-d \frac{\beta_{1}\left(S_{1}^{*}+a_{1} S_{1}^{* 2}\right)}{\left(1+a_{1} S_{1}^{*}+b_{1} I_{1}^{*}\right)^{2}} \\
= & \frac{\beta_{1}\left(I_{1}^{*}+b_{1} I_{1}^{* 2}\right)\left(d+\alpha_{1}\right)+d \beta_{1} S_{1}^{*} b_{1} I_{1}^{*}}{\left(1+a_{1} S_{1}^{*}+b_{1} I_{1}^{*}\right)^{2}}>0,
\end{aligned}
$$

then $\lambda_{2}$ and $\lambda_{3}$ have negative real parts, thus the equilibrium $E_{1}$ is stable. 
Similarly, we can show that if $\mathcal{R}_{1}<1$ and $\mathcal{R}_{2}>1$, then the equilibrium $E_{2}$ of system (3) is stable.

Now, let us prove that the positive equilibrium $E^{*}$ is stable as $\mathcal{R}_{1}>1$ and $\mathcal{R}_{2}>1$.

Denote the Jacobian matrix of (3) at the positive equilibrium $E^{*}$ by $J=\left(J_{i j}\right)$. Then $J_{i j}=\frac{\partial f_{i}}{\partial x_{j}}$, where $\left(x_{j}\right)=\left(S, I_{1}, I_{2}\right)$. More precisely,

$$
J^{*}=\left(\begin{array}{lll}
J_{11} & J_{12} & J_{13} \\
J_{21} & J_{22} & J_{23} \\
J_{31} & J_{32} & J_{33}
\end{array}\right),
$$

where

$$
\begin{aligned}
J_{11} & =-\frac{\beta_{1}\left(I_{1}^{* *}+b_{1} I_{1}^{* * 2}\right)}{\left(1+a_{1} S^{*}+b_{1} I_{1}^{* *}\right)^{2}}-\frac{\beta_{2}\left(I_{2}^{* *}+b_{2} I_{2}^{* * 2}\right)}{\left(1+a_{2} S^{*}+b_{2} I_{2}^{* *}\right)^{2}}-d \\
& =-J_{21}-J_{31}-d, \\
J_{12} & =r_{1}-\frac{\beta_{1}\left(S^{*}+a_{1} S^{* 2}\right)}{\left(1+a_{1} S^{*}+b_{1} I_{1}^{* *}\right)^{2}}=-\frac{\beta_{1}\left(S^{*}+a_{1} S^{* 2}\right)}{\left(1+a_{1} S^{*}+b_{1} I_{1}^{* *}\right)^{2}}-\left(d+\alpha_{1}\right) \\
& =-J_{22}-\left(d+\alpha_{1}\right), \\
J_{13} & =r_{2}-\frac{\beta_{2}\left(S^{*}+a_{2} S^{* 2}\right)}{\left(1+a_{2} S^{*}+b_{2} I_{2}^{* *}\right)^{2}}=-\frac{\beta_{2}\left(S^{*}+a_{2} S^{* 2}\right)}{\left(1+a_{2} S^{*}+b_{2} I_{2}^{* *}\right)^{2}}-\left(d+\alpha_{2}\right) \\
& =-J_{33}-\left(d+\alpha_{2}\right), \\
J_{21} & =\frac{\beta_{1}\left(I_{1}^{* *}+b_{1} I_{1}^{* * 2}\right)}{\left(1+a_{1} S^{*}+b_{1} I_{1}^{* *}\right)^{2}}, \\
J_{22} & =\frac{\beta_{1}\left(S^{*}+a_{1} S^{* 2}\right)}{\left(1+a_{1} S^{*}+b_{1} I_{1}^{* *}\right)^{2}}-\left(d+\alpha_{1}+r_{1}\right)=-\frac{\beta_{1} b_{1} S^{*} I_{1}^{* *}}{\left(1+a_{1} S^{*}+b_{1} I_{1}^{* *}\right)^{2}}, \\
J_{23} & =0, \\
J_{33} & =\frac{\beta_{2}\left(S^{*}+a_{2} S^{* 2}\right)}{\left(1+a_{2} S^{*}+b_{2} I_{2}^{* *}\right)^{2}}-\left(d+\alpha_{2}+r_{2}\right)=-\frac{\beta_{2} b_{2} S^{*} I_{2}^{* *}}{\left(1+a_{2} S^{*}+b_{2} I_{2}^{* *}\right)^{2}} . \\
J_{32} & =\frac{\beta_{2}\left(I_{2}^{* *}+b_{2} I_{2}^{* * 2}\right)}{\left(1+a_{2} S^{*}+b_{2} I_{2}^{* *}\right)^{2}}, \\
& 0,
\end{aligned}
$$

At the positive equilibrium $E^{*}$ we have the following characteristic equation:

$$
\lambda^{3}+p_{2} \lambda^{2}+p_{1} \lambda+p_{0}=0
$$

where

$$
\begin{aligned}
& p_{2}=-\left(J_{11}+J_{22}+J_{33}\right), \\
& p_{1}=J_{11} J_{22}+J_{11} J_{33}+J_{22} J_{33}-J_{13} J_{31}-J_{12} J_{21}, \\
& p_{0}=J_{12} J_{21} J_{33}+J_{13} J_{31} J_{22}-J_{11} J_{22} J_{33} .
\end{aligned}
$$

Then the equilibrium $E^{*}$ is stable if $p_{j}>0(j=1,2,3)$ and $p_{2} p_{1}>p_{0}$. 
Note that

$$
\begin{aligned}
& p_{2}=-\left(J_{11}+J_{22}+J_{33}\right) \\
& =J_{21}-J_{22}+J_{31}-J_{33}+d \\
& =\frac{\beta_{1} I_{1}^{* *}\left(1+b_{1} I_{1}^{* *}+b_{1} S^{*}\right)}{\left(1+a_{1} S^{*}+b_{1} I_{1}^{* *}\right)^{2}}+\frac{\beta_{2} I_{2}^{* *}\left(1+b_{2} I_{2}^{* *}+b_{2} S^{*}\right)}{\left(1+a_{2} S^{*}+b_{2} I_{2}^{* *}\right)^{2}}+d \\
& =p_{21}+p_{22}+d>0 \text {, } \\
& p_{1}=-\left(J_{31}+d\right) J_{22}-\left(J_{21}+d\right) J_{33}+J_{22} J_{33}+\left(d+\alpha_{2}\right) J_{31}+\left(d+\alpha_{1}\right) J_{21} \\
& =J_{22} J_{33}-J_{22} J_{31}-J_{21} J_{33}+J_{21}\left(d+\alpha_{1}\right)-d J_{22}+J_{31}\left(d+\alpha_{2}\right)-d J_{33} \\
& =\frac{\beta_{1} \beta_{2} S^{*} I_{1}^{* *} I_{2}^{* *}}{\left(1+a_{1} S^{*}+b_{1} I_{1}^{* *}\right)^{2}}\left(b_{1} b_{2} S^{*}+b_{1}\left(1+b_{2} I_{2}^{* *}\right)+b_{2}\left(1+b_{1} I_{1}^{* *}\right)\right) \\
& +\frac{\beta_{1} I_{1}^{* *}}{\left(1+a_{1} S^{*}+b_{1} I_{1}^{* *}\right)^{2}}\left(\left(1+b_{1} I_{1}^{* *}\right)\left(d+\alpha_{1}\right)+d b_{1} S^{*}\right) \\
& +\frac{\beta_{2} I_{2}^{* *}}{\left(1+a_{2} S^{*}+b_{2} I_{2}^{* *}\right)^{2}}\left(\left(1+b_{2} I_{2}^{* *}\right)\left(d+\alpha_{2}\right)+d b_{2} S^{*}\right) \\
& =p_{11}+p_{12}+p_{13}>0 \text {, } \\
& p_{0}=J_{12} J_{21} J_{33}+J_{13} J_{31} J_{22}-J_{11} J_{22} J_{33} \\
& =-\left(J_{22}+\left(d+\alpha_{1}\right)\right) J_{21} J_{33}-\left(J_{33}+\left(d+\alpha_{2}\right)\right) J_{31} J_{22}+\left(J_{21}+J_{31}+d\right) J_{22} J_{33} \\
& =-\left(d+\alpha_{1}\right) J_{21} J_{33}-\left(d+\alpha_{2}\right) J_{22} J_{31}+d J_{22} J_{33} \\
& =\left(d+\alpha_{1}\right) \frac{\beta_{1}\left(I_{1}^{* *}+b_{1} I_{1}^{* * 2}\right)}{\left(1+a_{1} S^{*}+b_{1} I_{1}^{* *}\right)^{2}} \frac{\beta_{2} b_{2} S^{*} I_{2}^{* *}}{\left(1+a_{2} S^{*}+b_{2} I_{2}^{* *}\right)^{2}} \\
& +\left(d+\alpha_{2}\right) \frac{\beta_{1} b_{1} S^{*} I_{1}^{* *}}{\left(1+a_{1} S^{*}+b_{1} I_{1}^{* *}\right)^{2}} \frac{\beta_{2}\left(I_{2}^{* *}+b_{2} I_{2}^{* * 2}\right)}{\left(1+a_{2} S^{*}+b_{2} I_{2}^{* *}\right)^{2}} \\
& +d \frac{\beta_{1} b_{1} S^{*} I_{1}^{* *}}{\left(1+a_{1} S^{*}+b_{1} I_{1}^{* *}\right)^{2}} \frac{\beta_{2} b_{2} S^{*} I_{2}^{* *}}{\left(1+a_{2} S^{*}+b_{2} I_{2}^{* *}\right)^{2}}>0 .
\end{aligned}
$$

Then we have

$$
\begin{aligned}
p_{2} p_{1}-p_{0}= & {\left[\left(p_{21}+p_{22}\right) p_{11}+p_{21} p_{12}+p_{22} p_{13}+d p_{1}\right] } \\
& +\left(p_{21} p_{13}+p_{22} p_{12}-p_{0}\right) \\
= & p+q .
\end{aligned}
$$

It is easy to see that $p>0$ when $\mathcal{R}_{1}>1$ and $\mathcal{R}_{2}>1$. Let us now verify that

$$
\begin{aligned}
q= & p_{21} p_{13}+p_{22} p_{12}-p_{0} \\
= & \frac{\beta_{1} I_{1}^{* *}\left(1+b_{1} I_{1}^{* *}+b_{1} S^{*}\right)}{\left(1+a_{1} S^{*}+b_{1} I_{1}^{* *}\right)^{2}} \frac{\beta_{2} I_{2}^{* *}}{\left(1+a_{2} S^{*}+b_{2} I_{2}^{* *}\right)^{2}}\left(\left(1+b_{2} I_{2}^{* *}\right)\left(d+\alpha_{2}\right)+d b_{2} S^{*}\right) \\
& +\frac{\beta_{2} I_{2}^{* *}\left(1+b_{2} I_{2}^{* *}+b_{2} S^{*}\right)}{\left(1+a_{2} S^{*}+b_{2} I_{2}^{* *}\right)^{2}} \frac{\beta_{1} I_{1}^{* *}}{\left(1+a_{1} S^{*}+b_{1} I_{1}^{* *}\right)^{2}}\left(\left(1+b_{1} I_{1}^{* *}\right)\left(d+\alpha_{1}\right)+d b_{1} S^{*}\right) \\
& -\left(d+\alpha_{1}\right) \frac{\beta_{1}\left(I_{1}^{* *}+b_{1} I_{1}^{* * 2}\right)}{\left(1+a_{1} S^{*}+b_{1} I_{1}^{* *}\right)^{2}} \frac{\beta_{2} b_{2} S^{*} I_{2}^{* *}}{\left(1+a_{2} S^{*}+b_{2} I_{2}^{* *}\right)^{2}}
\end{aligned}
$$




$$
\begin{aligned}
& -\left(d+\alpha_{2}\right) \frac{\beta_{1} b_{1} S^{*} I_{1}^{* *}}{\left(1+a_{1} S^{*}+b_{1} I_{1}^{* *}\right)^{2}} \frac{\beta_{2}\left(I_{2}^{* *}+b_{2} I_{2}^{* * 2}\right)}{\left(1+a_{2} S^{*}+b_{2} I_{2}^{* *}\right)^{2}} \\
& -d \frac{\beta_{1} b_{1} S^{*} I_{1}^{* *}}{\left(1+a_{1} S^{*}+b_{1} I_{1}^{* *}\right)^{2}} \frac{\beta_{2} b_{2} S^{*} I_{2}^{* *}}{\left(1+a_{2} S^{*}+b_{2} I_{2}^{* *}\right)^{2}} \\
& =\frac{\beta_{1} \beta_{2} I_{1}^{* *} I_{2}^{* *}}{\left(1+a_{1} S^{*}+b_{1} I_{1}^{* *}\right)^{2}\left(1+a_{2} S^{*}+b_{2} I_{2}^{* *}\right)^{2}}\left(( 1 + b _ { 1 } I _ { 1 } ^ { * * } ) \left(d+\alpha_{2}+d b_{2} I_{2}^{* *}\right.\right. \\
& \left.\quad+d b_{2} S^{*}+\alpha_{2} b_{2} I_{2}^{* *}\right)+\left(1+b_{2} I_{2}^{* *}\right)\left(d+\alpha_{1}+d b_{1} I_{1}^{* *}+\alpha_{1} b_{1} I_{1}^{* *}+d b_{1} S^{*}\right. \\
& \left.\left.\quad+d b_{1} b_{2} S^{* 2}\right)\right)>0,
\end{aligned}
$$

which implies that $p_{2} p_{1}>p_{0}$. Then it can be concluded that $E^{*}$ is stable when it exists. The proof is completed.

\section{Dynamics of stochastic system (4)}

\subsection{Extinction}

In this section, we are going to explore the conditions which lead to the extinction of two infectious diseases mentioned in the system (4) under a white noise stochastic disturbance.

\section{Theorem 4.1 If}

$$
\sigma_{i}>\frac{\beta_{i}}{\sqrt{2\left(d+\alpha_{i}+r_{i}\right)}}, \quad i=1,2,
$$

then two infectious diseases of system (4) go to extinction almost surely.

Proof Let $\left(S(t), I_{1}(t), I_{2}(t)\right)$ be a solution of system (4) with initial value $\left(S(0), I_{1}(0), I_{2}(0)\right) \in$ $R_{+}^{3}$. Applying Itô's formula to system (4) results in

$$
\begin{aligned}
\mathrm{d} \ln I_{i}(t)= & \left(\frac{\beta_{i} S(t)}{1+a_{i} S(t)+b_{i} I_{i}(t)}-\left(d+\alpha_{i}+r_{i}\right)-\frac{\sigma_{i}^{2} S^{2}(t)}{2\left(1+a_{i} S(t)+b_{i} I_{i}(t)\right)^{2}}\right) \mathrm{d} t \\
& +\frac{\sigma_{i} S(t)}{1+a_{i} S(t)+b_{i} I_{i}(t)} \mathrm{d} B_{i}(t), \quad i=1,2 .
\end{aligned}
$$

Integrating both sides of (9) from 0 to $t$ gives

$$
\begin{aligned}
\ln I_{i}(t)= & -\frac{\sigma_{i}^{2}}{2} \int_{0}^{t}\left(\frac{S(\tau)}{1+a_{i} S(\tau)+b_{i} I_{i}(\tau)}-\frac{\beta_{i}}{\sigma_{i}^{2}}\right)^{2} \mathrm{~d} \tau-\left(d+\alpha_{i}+r_{i}\right) t \\
& +\frac{\beta_{i}^{2}}{2 \sigma_{i}^{2}} t+M_{i}(t)+\ln I_{i}(0) \\
\leq & -\left(d+\alpha_{i}+r_{i}\right) t+\frac{\beta_{i}^{2}}{2 \sigma_{i}^{2}} t+M_{i}(t)+\ln I_{i}(0),
\end{aligned}
$$

where $M_{i}(t)=\int_{0}^{t} \frac{\sigma_{i} S(\tau)}{1+a_{i} S(\tau)+b_{i} I_{i}(\tau)} \mathrm{d} B_{i}(\tau), i=1,2$.

Dividing both sides of (10) by $t$, we have

$$
\frac{\ln I_{i}(t)}{t} \leq-\left(d+\alpha_{i}+r_{i}-\frac{\beta_{i}^{2}}{2 \sigma_{i}^{2}}\right)+\frac{M_{i}(t)}{t}+\frac{\ln I_{i}(0)}{t}, \quad i=1,2 .
$$


The function $M_{i}(t)(i=1,2)$ is also known as the local continuous martingale with $M_{i}(0)=$ 0 , and by Lemma 2.3 , we have

$$
\lim _{t \rightarrow+\infty} \frac{M_{i}(t)}{t}=0, \quad i=1,2
$$

Since $\sigma_{i}>\frac{\beta_{i}}{\sqrt{2\left(d+\alpha_{i}+r_{i}\right)}}$ for $i=1,2$, taking the limit superior of both sides of (11) leads to

$$
\limsup _{t \rightarrow+\infty} \frac{\ln I_{i}(t)}{t} \leq-\left(d+\alpha_{i}+r_{i}-\frac{\beta_{i}^{2}}{2 \sigma_{i}^{2}}\right)<0
$$

which implies that $\lim _{t \rightarrow+\infty} I_{i}(t)=0$. This completes the proof of Theorem 4.1.

Remark 4.1 Theorem 4.1 shows that when $\sigma_{i}>\frac{\beta_{i}}{\sqrt{2\left(d+\alpha_{i}+r_{i}\right)}}, i=1,2$, two infectious diseases of system (4) die out almost surely, that is to say, large white noise stochastic disturbance can lead to the two epidemics to be extinct. Therefore, we always assume that the white noise stochastic disturbance is not too large in the rest of this paper.

Let

$$
\begin{aligned}
\mathcal{R}_{1}^{*} & =\frac{\beta_{1} A}{\left(A a_{1}+d\right)\left(d+\alpha_{1}+r_{1}\right)}-\frac{\sigma_{1}^{2} A^{2}}{2\left(A a_{1}+d\right)^{2}\left(d+\alpha_{1}+r_{1}\right)} \\
& =\mathcal{R}_{1}-\frac{\sigma_{1}^{2} A^{2}}{2\left(A a_{1}+d\right)^{2}\left(d+\alpha_{1}+r_{1}\right)}, \\
\mathcal{R}_{2}^{*} & =\frac{\beta_{2} A}{\left(A a_{2}+d\right)\left(d+\alpha_{2}+r_{2}\right)}-\frac{\sigma_{2}^{2} A^{2}}{2\left(A a_{2}+d\right)^{2}\left(d+\alpha_{2}+r_{2}\right)} \\
& =\mathcal{R}_{2}-\frac{\sigma_{2}^{2} A^{2}}{2\left(A a_{2}+d\right)^{2}\left(d+\alpha_{2}+r_{2}\right)},
\end{aligned}
$$

where $\mathcal{R}_{1}$ and $\mathcal{R}_{2}$ are the threshold of the deterministic system (3) given in (8). Then we have the following results mentioned in the theorem.

Theorem 4.2 Let $\left(S(t), I_{1}(t), I_{2}(t)\right)$ be a solution of system (4) with initial value $\left(S(0), I_{1}(0)\right.$, $\left.I_{2}(0)\right) \in R_{+}^{3}$. Then if

$$
\mathcal{R}_{i}^{*}<1 \quad \text { and } \quad \sigma_{i} \leq \sqrt{\frac{\beta_{i}\left(A a_{i}+d\right)}{A}}, \quad i=1,2
$$

hold, two infectious diseases of system (4) go to extinction almost surely, i.e.

$$
\lim _{t \rightarrow+\infty} I_{i}(t)=0, \quad i=1,2 .
$$

Moreover, $\lim _{t \rightarrow+\infty} S(t)=\frac{A}{d}$, almost surely. 
Proof For both sides of (9), integrating from 0 to $t$ first and dividing by $t$ yields

$$
\begin{aligned}
\frac{\ln I_{i}(t)}{t}= & \frac{1}{t} \int_{0}^{t}\left(\frac{\beta_{i} S(\tau)}{1+a_{i} S(t)+b_{i} I_{i}(t)}-\left(d+\alpha_{i}+r_{i}\right)\right. \\
& \left.-\frac{\sigma_{i}^{2} S^{2}(\tau)}{2\left(1+a_{i} S(t)+b_{i} I_{i}(t)\right)^{2}}\right) \mathrm{d} \tau+\frac{M_{i}(t)}{t}+\frac{\ln I_{i}(0)}{t} \\
\leq & \left(\frac{\beta_{i} A}{A a_{i}+d}-\left(d+\alpha_{i}+r_{i}\right)-\frac{\sigma_{i}^{2} A^{2}}{2\left(A a_{i}+d\right)^{2}}\right)+\frac{M_{i}(t)}{t}+\frac{\ln I_{i}(0)}{t} \\
= & \left(d+\alpha_{i}+r_{i}\right)\left(\frac{\beta_{i} A}{\left(A a_{i}+d\right)\left(d+\alpha_{i}+r_{i}\right)}-\frac{\sigma_{i}^{2}}{2\left(A a_{i}+d\right)^{2}\left(d+\alpha_{i}+r_{i}\right)}-1\right) \\
& +\frac{M_{i}(t)}{t}+\frac{\ln I_{i}(0)}{t} .
\end{aligned}
$$

Taking the superior limit of both sides of (12) leads to

$$
\limsup _{t \rightarrow+\infty} \frac{\ln I_{i}(t)}{t} \leq\left(d+\alpha_{i}+r_{i}\right)\left(\mathcal{R}_{i}^{*}-1\right)<0
$$

which implies that $\lim _{t \rightarrow+\infty} I_{i}(t)=0, i=1,2$.

Without loss of generality, we assume that $0<I_{i}(t)<\varepsilon_{i}(i=1,2)$ for all $t \geq 0$, by the first equation of system (4), we have

$$
\frac{\mathrm{d} S(t)}{\mathrm{d} t} \geq A-\left(d+\beta_{1} \varepsilon_{1}+\beta_{2} \varepsilon_{2}+\sigma_{1} \varepsilon_{1}\left|\dot{B}_{1}(t)\right|+\sigma_{2} \varepsilon_{2}\left|\dot{B}_{2}(t)\right|\right) S(t) .
$$

As $\varepsilon_{1} \rightarrow 0$ and $\varepsilon_{2} \rightarrow 0$, taking the inferior limit of both sides of (13) yields

$$
\liminf _{t \rightarrow+\infty} S(t) \geq \frac{A}{d}
$$

By the proof of Lemma 2.2, we have

$$
\limsup _{t \rightarrow+\infty} S(t) \leq \frac{A}{d}
$$

From (14) and (15), we have

$$
\lim _{t \rightarrow+\infty} S(t)=\frac{A}{d}
$$

almost surely. This completes the proof of Theorem 4.2.

Remark 4.2 Theorem 4.1 and Theorem 4.2 show that two diseases will die out if the white noise disturbance is sufficiently larger or $\mathcal{R}_{i}^{*}<1$ and the white noise disturbance is not large. Note that the expressions for $\mathcal{R}_{i}^{*}$ for $i=1,2$ which are the threshold values of system (4) are strictly different compared with the thresholds $\mathcal{R}_{i}$ of system (3), This implies that the conditions which are needed to have $I_{i}(t)$ for $i=1,2$ gone in extinction in deterministic system (3) are stronger than in the corresponding stochastic system (4). 


\subsection{Permanence in mean}

Theorem 4.3 Let $\left(S(t), I_{1}(t), I_{2}(t)\right)$ be the solution of system (4) with initial value $\left(S(0), I_{1}(0)\right.$, $\left.I_{2}(0)\right) \in \Gamma$, then we have the following.

(i) If $\mathcal{R}_{1}^{*}>1, \mathcal{R}_{2}^{*}<1$ and $\sigma_{2} \leq \sqrt{\frac{\beta_{2}\left(A a_{2}+d\right)}{A}}$, then the disease $I_{2}$ goes extinct and the disease $I_{1}$ is permanent in mean, moreover, $I_{1}$ satisfies

$$
\liminf _{t \rightarrow+\infty}\left\langle I_{1}(t)\right\rangle \geq \frac{\left(A a_{1}+d\right)\left(d+\alpha_{1}+r_{1}\right)}{\beta_{1}\left(d+\alpha_{1}\right)+b_{1} d\left(d+\alpha_{1}+r_{1}\right)}\left(\mathcal{R}_{1}^{*}-1\right) .
$$

(ii) If $\mathcal{R}_{2}^{*}>1, \mathcal{R}_{1}^{*}<1$ and $\sigma_{1} \leq \sqrt{\frac{\beta_{1}\left(A a_{1}+d\right)}{A}}$, then the disease $I_{1}$ goes extinct and the disease $I_{2}$ is permanent in mean, moreover, $I_{2}$ satisfies

$$
\liminf _{t \rightarrow+\infty}\left\langle I_{2}(t)\right| \geq \frac{\left(A a_{2}+d\right)\left(d+\alpha_{2}+r_{2}\right)}{\beta_{2}\left(d+\alpha_{2}\right)+b_{2} d\left(d+\alpha_{2}+r_{2}\right)}\left(\mathcal{R}_{2}^{*}-1\right)
$$

(iii) If $\mathcal{R}_{1}^{*}>1$ and $\mathcal{R}_{2}^{*}>1$, then two infectious diseases $I_{1}$ and $I_{2}$ are permanent in mean, moreover, $I_{1}$ and $I_{2}$ satisfy

$$
\liminf _{t \rightarrow+\infty}\left\langle I_{1}(t)+I_{2}(t)\right\rangle \geq \frac{1}{\Delta_{\max }} \sum_{i=1}^{2} a_{i}\left(d+\alpha_{i}+r_{i}\right)\left(\mathcal{R}_{i}^{*}-1\right),
$$

where

$$
\Delta_{\max }=\sum_{i=1}^{2}\left[\frac{\beta_{1}+\beta_{2}}{d}\left(d+\alpha_{i}\right)+b_{i}\left(d+\alpha_{i}+r_{i}\right)\right]
$$

Proof Part (i). By Theorem 3.1, since $\mathcal{R}_{2}^{*}<1$ and $\sigma_{2} \leq \sqrt{\frac{\beta_{2}\left(A a_{2}+d\right)}{A}}$, we have $\lim _{t \rightarrow+\infty} I_{2}(t)=0$. Since $\mathcal{R}_{1}^{*}>1$, for $\varepsilon$ small enough, such that $0<I_{2}(t)<\varepsilon$ for all $t$ large enough we have

$$
\frac{\beta_{1}\left(A-\left(d+\alpha_{1}\right) \varepsilon\right)}{\left(A a_{1}+d\right)\left(d+\alpha_{1}+r_{1}\right)}-\frac{\sigma_{1}^{2} A^{2}}{2\left(A a_{1}+d\right)^{2}\left(d+\alpha_{1}+r_{1}\right)}>1
$$

Integrating from 0 to $t$ and dividing by $t>0$ on both sides of system (4) yields

$$
\begin{aligned}
\Theta(t) & \triangleq \frac{S(t)-S(0)}{t}+\frac{I_{1}(t)-I_{1}(0)}{t}+\frac{I_{2}(t)-I_{2}(0)}{t} \\
& =A-d\langle S(t)\rangle-\left(d+\alpha_{1}\right)\left\langle I_{1}(t)\right\rangle-\left(d+\alpha_{2}\right)\left\langle I_{2}(t)\right\rangle \\
& \geq A-d\langle S(t)\rangle-\left(d+\alpha_{1}\right)\left\langle I_{1}(t)\right\rangle-\left(d+\alpha_{2}\right) \varepsilon,
\end{aligned}
$$

then one can get

$$
\langle S(t)\rangle \geq \frac{A-\left(d+\alpha_{2}\right) \varepsilon}{d}-\frac{d+\alpha_{1}}{d}\left\langle I_{1}(t)\right\rangle-\frac{\Theta(t)}{d} .
$$


Applying Itô's formula gives

$$
\begin{aligned}
\mathrm{d}( & \left.\left(1+a_{1} \frac{A}{d}\right) \ln I_{1}(t)+b_{1} I_{1}(t)\right) \\
= & {\left[\frac{\left(1+a_{1} \frac{A}{d}\right) \beta_{1} S(t)}{1+a_{1} S(t)+b_{1} I_{1}(t)}-\left(1+a_{1} \frac{A}{d}\right)\left(d+\alpha_{1}+r_{1}\right)-\frac{\left(1+a_{1} \frac{A}{d}\right) \sigma_{1}^{2} S^{2}(t)}{1+a_{1} S(t)+b_{1} I_{1}(t)}\right] \mathrm{d} t } \\
& +b_{1}\left[\frac{\beta_{1} S(t) I_{1}(t)}{1+a_{1} S(t)+b_{1} I_{1}(t)}-\left(d+\alpha_{1}+r_{1}\right) I_{1}(t)\right] \mathrm{d} t \\
& +\frac{\left(1+a_{1} \frac{A}{d}\right) \sigma_{1} S(t) \mathrm{d} B_{1}(t)}{1+a_{1} S(t)+b_{1} I_{1}(t)}+\frac{b_{1} \sigma_{1} S(t) I_{1}(t) \mathrm{d} B_{1}(t)}{1+a_{1} S(t)+b_{1} I_{1}(t)} \\
\geq & \left.\frac{\left(1+a_{1} \frac{A}{d}\right) \beta_{1} S(t)}{1+a_{1} \frac{A}{d}+b_{1} I_{1}(t)}-\left(1+a_{1} \frac{A}{d}\right)\left(d+\alpha_{1}+r_{1}\right)-\frac{\left(1+a_{1} \frac{A}{d}\right) \sigma_{1}^{2} S^{2}(t)}{1+a_{1} S(t)+b_{1} I_{1}(t)}\right] \mathrm{d} t \\
& +b_{1}\left[\frac{\beta_{1} S(t) I_{1}(t)}{1+a_{1} \frac{A}{d}+b_{1} I_{1}(t)}-\left(d+\alpha_{1}+r_{1}\right) I_{1}(t)\right] \mathrm{d} t \\
& +\frac{\left(1+a_{1} \frac{A}{d}\right) \sigma_{1} S(t) \mathrm{d} B_{1}(t)}{1+a_{1} \frac{A}{d}+b_{1} I_{1}(t)}+\frac{b_{1} \sigma_{1} S(t) I_{1}(t) \mathrm{d} B_{1}(t)}{1+a_{1} \frac{A}{d}+b_{1} I_{1}(t)} \\
\geq & {\left[\beta_{1} S(t)-\left(1+a_{1} \frac{A}{d}\right)\left(d+\alpha_{1}+r_{1}\right)-b_{1}\left(d+\alpha_{1}+r_{1}\right) I_{1}(t)-\frac{\sigma_{1}^{2}\left(\frac{A}{d}\right)^{2}}{2\left(1+a_{1} \frac{A}{d}\right)}\right] \mathrm{d} t } \\
& +\sigma_{1} S(t) \mathrm{d} B_{1}(t) .
\end{aligned}
$$

Integrating from 0 to $t$ and dividing by $t>0$ on both sides of (16) yields

$$
\begin{aligned}
&\left(1+a_{1} \frac{A}{d}\right) \frac{\left(\ln I_{1}(t)-\ln I_{1}(0)\right)}{t}+b_{1} \frac{I_{1}(t)-I_{1}(0)}{t} \\
& \geq \beta_{1}\langle S(t)\rangle-\left(1+a_{1} \frac{A}{d}\right)\left(d+\alpha_{1}+r_{1}\right)-b_{1}\left(d+\alpha_{1}+r_{1}\right)\left\langle I_{1}(t)\right\rangle \\
& \quad-\frac{\sigma_{1}^{2}\left(\frac{A}{d}\right)^{2}}{2\left(1+a_{1} \frac{A}{d}\right)}+\frac{M(t)}{t} \\
& \geq \beta_{1}\left[\frac{A-\left(d+\alpha_{2}\right) \varepsilon}{d}-\frac{d+\alpha_{1}}{d}\left\langle I_{1}(t)\right\rangle-\frac{\Theta(t)}{d}\right] \\
&-\left(1+a_{1} \frac{A}{d}\right)\left(d+\alpha_{1}+r_{1}\right) \\
&-b_{1}\left(d+\alpha_{1}+r_{1}\right)\left\langle I_{1}(t)\right\rangle-\frac{\sigma_{1}^{2}\left(\frac{A}{d}\right)^{2}}{2\left(1+a_{1} \frac{A}{d}\right)}+\frac{M(t)}{t} \\
&=\left(1+a_{1} \frac{A}{d}\right)\left(d+\alpha_{1}+r_{1}\right)\left[\frac{\beta_{1}\left(A-\left(d+\alpha_{2}\right) \varepsilon\right)}{d\left(1+a_{1} \frac{A}{d}\right)\left(d+\alpha_{1}+r_{1}\right)}\right. \\
&\left.-\frac{\sigma_{1}^{2}\left(\frac{A}{d}\right)^{2}}{2\left(1+a_{1} \frac{A}{d}\right)^{2}\left(d+\alpha_{1}+r_{1}\right)}-1\right]-\left[\frac{\beta_{1}\left(d+\alpha_{1}\right)}{d}+b_{1}\left(d+\alpha_{1}+r_{1}\right)\right]\left\langle I_{1}(t)\right\rangle \\
&-\frac{M(t)}{d}+\frac{\beta_{1}}{t},
\end{aligned}
$$


where $M(t)=\int_{0}^{t} \sigma_{1} S(\tau) \mathrm{d} B_{1}(\tau)$. The inequality (17) can be rewritten as

$$
\begin{aligned}
\left\langle I_{1}(t)\right\rangle \geq & \frac{1}{\Delta}\left[( 1 + a _ { 1 } \frac { A } { d } ) ( d + \alpha _ { 1 } + r _ { 1 } ) \left(\frac{\beta_{1}\left(A-\left(d+\alpha_{2}\right) \varepsilon\right)}{d\left(1+a_{1} \frac{A}{d}\right)\left(d+\alpha_{1}+r_{1}\right)}\right.\right. \\
& \left.-\frac{\sigma_{1}^{2}\left(\frac{A}{d}\right)^{2}}{2\left(1+a_{1} \frac{A}{d}\right)^{2}\left(d+\alpha_{1}+r_{1}\right)}-1\right)-\frac{\beta_{1} \Theta(t)}{d}+\frac{M(t)}{t} \\
& \left.-\left(1+a_{1} \frac{A}{d}\right) \frac{\left(\ln I_{1}(t)-\ln I_{1}(0)\right)}{t}-b_{1} \frac{I_{1}(t)-I_{1}(0)}{t}\right] \\
\geq & \left\{\begin{array}{l}
\frac{1}{\Delta}\left[( 1 + a _ { 1 } \frac { A } { d } ) ( d + \alpha _ { 1 } + r _ { 1 } ) \left(\frac{\beta_{1}\left(A-\left(d+\alpha_{2}\right) \varepsilon\right)}{d\left(1+a_{1} \frac{A}{d}\right)\left(d+\alpha_{1}+r_{1}\right)}\right.\right. \\
\left.\quad-\frac{\sigma_{1}^{2}\left(\frac{A}{d}\right)^{2}}{2\left(1+a_{1} \frac{A}{d}\right)^{2}\left(d+\alpha_{1}+r_{1}\right)}-1\right)-\frac{\beta_{1} \Theta(t)}{d}+\frac{M(t)}{t} \\
\left.\quad+\left(1+a_{1} \frac{A}{d}\right) \frac{\ln I_{1}(0)}{t}-b_{1} \frac{I_{1}(t)-I_{1}(0)}{t}\right], \\
\frac{1}{\Delta}\left[a _ { 1 } ( d + \alpha _ { 1 } + r _ { 1 } ) \left(\frac{\beta_{1}\left(A-\left(d+\alpha_{2}\right) \varepsilon\right)}{d\left(1+a_{1} \frac{A}{d}\right)\left(d+\alpha_{1}+r_{1}\right)}\right.\right. \\
\left.\quad-\frac{\sigma_{1}^{2}\left(\frac{A}{d}\right)^{2}}{2\left(1+a_{1} \frac{A}{d}\right)^{2}\left(d+\alpha_{1}+r_{1}\right)}-1\right)-\frac{\beta_{1} \Theta(t)}{d}+\frac{M(t)}{t} \\
\left.-\left(1+a_{1} \frac{A}{d}\right) \frac{\left(\ln I_{1}(t)-\ln I_{1}(0)\right)}{t}-b_{1} \frac{I_{1}(t)-I_{1}(0)}{t}\right], \quad 1 \leq I_{1}(t),
\end{array}\right.
\end{aligned}
$$

where $\Delta=\frac{\beta_{1}\left(d+\alpha_{1}\right)}{d}+b_{1}\left(d+\alpha_{1}+r_{1}\right)$.

By Lemma 2.3, we get $\lim _{t \rightarrow+\infty} \frac{M(t)}{t}=0$. According to Lemma 2.2, one can see that $I_{1}(t) \leq \frac{A}{d}$. Thus, one has $\lim _{t \rightarrow+\infty} \frac{I_{1}(t)}{t}=0, \lim _{t \rightarrow+\infty} \frac{\ln I_{1}(t)}{t}=0$ as $I_{1}(t) \geq 1$ and $\lim _{t \rightarrow+\infty} \Theta(t)=0$.

Taking the inferior limit of both sides of (18) yields

$$
\begin{aligned}
\liminf _{t \rightarrow+\infty}\left|I_{1}(t)\right\rangle \geq & \frac{\left(1+a_{1} \frac{A}{d}\right)\left(d+\alpha_{1}+r_{1}\right)}{\Delta}\left[\frac{\beta_{1}\left(A-\left(d+\alpha_{2}\right) \varepsilon\right)}{\left(d+a_{1} A\right)\left(d+\alpha_{1}+r_{1}\right)}\right. \\
& \left.-\frac{\sigma_{1}^{2} A^{2}}{2\left(d+a_{1} A\right)^{2}\left(d+\alpha_{1}+r_{1}\right)}-1\right] \\
> & 0 .
\end{aligned}
$$

Letting $\varepsilon \rightarrow 0$, we have

$$
\liminf _{t \rightarrow+\infty}\left\{I_{1}(t)\right\rangle \geq \frac{d\left(1+a_{1} \frac{A}{d}\right)\left(d+\alpha_{1}+r_{1}\right)}{\beta_{1}\left(d+\alpha_{1}\right)+b_{1} d\left(d+\alpha_{1}+r_{1}\right)}\left(\mathcal{R}_{1}^{*}-1\right) .
$$

Similarly, by using arguments as in the part (i), we can establish the results given in part (ii), and we omit it here.

Part (iii). Notice that

$$
\langle S(t)\rangle=\frac{A}{d}-\frac{d+\alpha_{1}}{d}\left\langle I_{1}(t)\right\rangle-\frac{d+\alpha_{2}}{d}\left\langle I_{2}(t)\right\rangle-\frac{\Theta(t)}{d} .
$$

Define

$$
V(t)=\ln \left[I_{1}^{\left(1+a_{1} \frac{A}{d}\right)}(t) I_{2}^{\left(1+a_{2} \frac{A}{d}\right)}(t)\right]+\left[b_{1} I_{1}(t)+b_{2} I_{2}(t)\right] .
$$


Therefore, $V(t)$ is bounded. Then we have

$$
\begin{aligned}
& D^{+} V(t)=\sum_{i=1}^{2}\left[\frac{\left(1+a_{i} \frac{A}{d}\right) \beta_{i} S(t)}{1+a_{i} S(t)+b_{i} I_{i}(t)}-\left(1+a_{i} \frac{A}{d}\right)\left(d+\alpha_{i}+r_{i}\right)\right. \\
& \left.-\frac{\left(1+a_{i} \frac{A}{d}\right) \sigma_{i}^{2} S^{2}(t)}{2\left(1+a_{i} S(t)+b_{i} I_{i}(t)\right)^{2}}\right] \mathrm{d} t+\sum_{i=1}^{2} \frac{\left(1+a_{i} \frac{A}{d}\right) \sigma_{i} S(t) \mathrm{d} B_{i}(t)}{1+a_{i} S(t)+b_{i} I_{i}(t)} \\
& +\sum_{i=1}^{2} b_{i}\left[\frac{\beta_{i} S(t) I_{i}(t)}{1+a_{i} S(t)+b_{i} I_{i}(t)}-\left(d+\alpha_{i}+r_{i}\right) I_{i}(t)\right] \mathrm{d} t \\
& +\sum_{i=1}^{2} \frac{b_{i} \sigma_{i} S(t) I_{i}(t) \mathrm{d} B_{i}(t)}{1+a_{i} S(t)+b_{i} I_{i}(t)} \\
& \geq \sum_{i=1}^{2}\left[\frac{\left(1+a_{i} \frac{A}{d}\right) \beta_{i} S(t)}{1+a_{i} \frac{A}{d}+b_{i} I_{i}(t)}-\left(1+a_{i} \frac{A}{d}\right)\left(d+\alpha_{i}+r_{i}\right)\right. \\
& \left.-\frac{\left(1+a_{i} \frac{A}{d}\right) \sigma_{i}^{2} S^{2}(t)}{2\left(1+a_{i} S(t)+b_{i} I_{i}(t)\right)^{2}}\right] \mathrm{d} t+\sum_{i=1}^{2} \frac{\left(1+a_{i} \frac{A}{d}\right) \sigma_{i} S(t) \mathrm{d} B_{i}(t)}{1+a_{i} \frac{A}{d}+b_{i} I_{i}(t)} \\
& +\sum_{i=1}^{2} b_{i}\left[\frac{\beta_{i} S(t) I_{i}(t)}{1+a_{i} \frac{A}{d}+b_{i} I_{i}(t)}-\left(d+\alpha_{i}+r_{i}\right) I_{i}(t)\right] \mathrm{d} t \\
& +\sum_{i=1}^{2} \frac{b_{i} \sigma_{i} S(t) I_{i}(t) \mathrm{d} B_{i}(t)}{1+a_{i} \frac{A}{d}+b_{i} I_{i}(t)} \\
& \geq\left[\left(\beta_{1}+\beta_{2}\right) S(t)-\sum_{i=1}^{2}\left(d+\alpha_{i}+r_{i}\right)\left(1+a_{1} \frac{A}{d}+b_{i} I_{i}(t)\right)\right. \\
& \left.-\sum_{i=1}^{2} \frac{\sigma_{i}^{2}\left(\frac{A}{d}\right)^{2}}{2\left(1+a_{i} \frac{A}{d}\right)}\right] \mathrm{d} t+\sum_{i=1}^{2} \sigma_{i} S(t) \mathrm{d} B_{i}(t) .
\end{aligned}
$$

Integrating from 0 to $t$ and dividing by $t>0$ on both sides of (20) yields

$$
\begin{aligned}
\frac{V(t)}{t}-\frac{V(0)}{t} \geq & {\left[\left(\beta_{1}+\beta_{2}\right)\langle S(t)\rangle-\sum_{i=1}^{2}\left(d+\alpha_{i}+r_{i}\right)\left(1+a_{i} \frac{A}{d}+b_{i}\left\langle I_{i}(t)\right\rangle\right)\right.} \\
& \left.-\sum_{i=1}^{2} \frac{\sigma_{i}^{2}\left(\frac{A}{d}\right)^{2}}{2\left(1+a_{i} \frac{A}{d}\right)}\right] \mathrm{d} t+\sum_{i=1}^{2} \frac{M_{i}}{t} \\
= & \left(\beta_{1}+\beta_{2}\right) \frac{A}{d}-\sum_{i=1}^{2}\left(d+\alpha_{i}+r_{i}\right)\left(1+a_{1} \frac{A}{d}\right)-\sum_{i=1}^{2} \frac{\sigma_{i}^{2}\left(\frac{A}{d}\right)^{2}}{2\left(1+a_{i} \frac{A}{d}\right)} \\
& -\sum_{i=1}^{2}\left[\frac{\beta_{1}+\beta_{2}}{d}\left(d+\alpha_{i}\right)+b_{i}\left(d+\alpha_{i}+r_{i}\right)\right]\left\langle I_{i}(t)\right\rangle \\
& -\frac{\beta_{1}+\beta_{2}}{d} \Theta(t)+\sum_{i=1}^{2} \frac{M_{i}}{t} \\
\geq & \sum_{i=1}^{2}\left(1+a_{i} \frac{A}{d}\right)\left(d+\alpha_{i}+r_{i}\right)\left[\frac{\beta_{i} A}{\left(d+a_{i} A\right)\left(d+\alpha_{i}+r_{i}\right)}\right.
\end{aligned}
$$


(a)

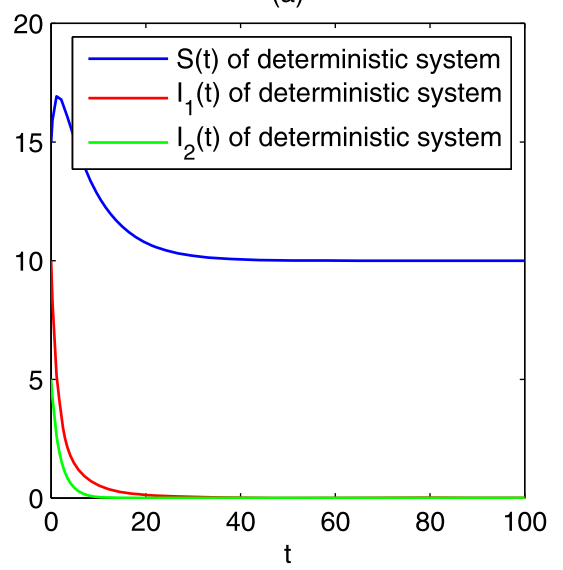

(c)

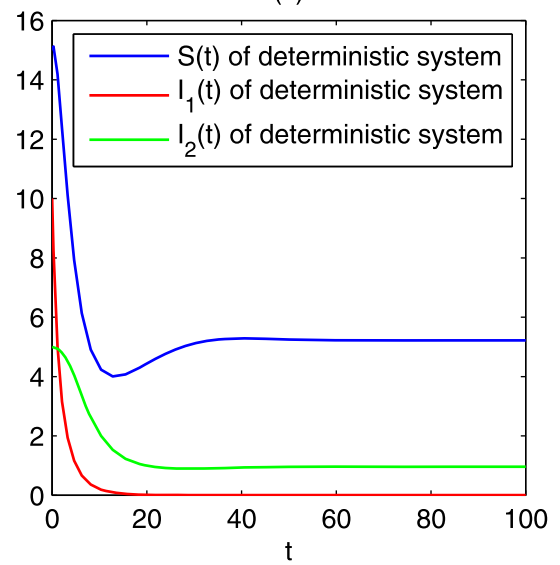

(b)

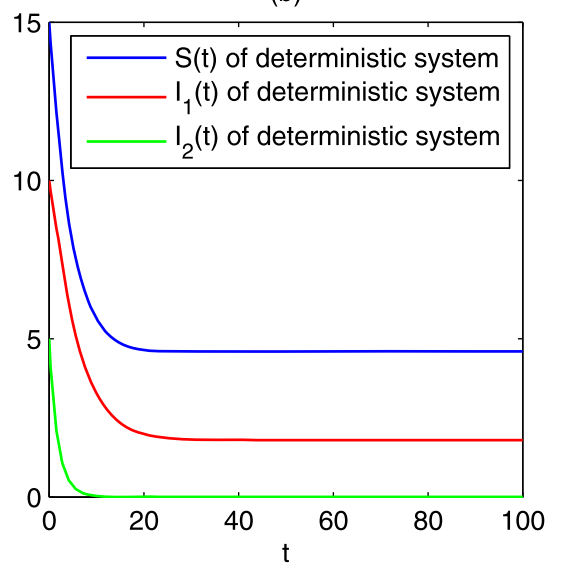

(d)

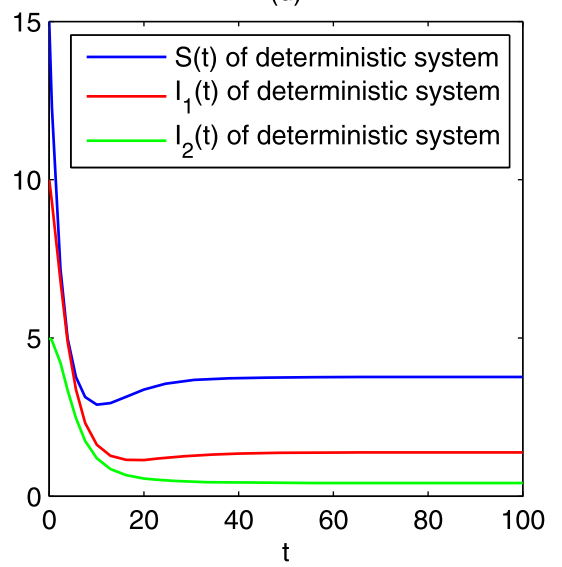

Figure 1 Time evolutions of the deterministic SIS system with parameters $A=1, d=0.1, \beta_{1}=1.2$, $\beta_{2}=1.5, a_{1}=1, a_{2}=1.5, b_{1}=2, b_{2}=1, \alpha_{1}=0.2, \alpha_{2}=0.4$. (a) Time series for $S(t), l_{1}(t), l_{2}(t)$ with parameters $r_{1}=0.9, r_{2}=0.9, R_{1}=0.9091, R_{2}=0.6696$. (b) Time series for $S(t), l_{1}(t), l_{2}(t)$ with parameters $r_{1}=0.3, r_{2}=0.9$, $R_{1}=1.8182, R_{2}=0.6696$. (c) Time series for $S(t), l_{1}(t), I_{2}(t)$ with parameters $r_{1}=0.9, r_{2}=0.3, R_{1}=0.9091$, $R_{2}=1.1719$. (d) Time series for $S(t), l_{1}(t), l_{2}(t)$ with parameters $r_{1}=0.3, r_{2}=0.3, R_{1}=1.8182, R_{2}=1.1719$.

$$
\begin{aligned}
& \left.-\frac{\sigma_{i}^{2} A^{2}}{2\left(d+a_{i} A\right)^{2}\left(d+\alpha_{i}+r_{i}\right)}-1\right]-\Delta_{\max }\left[\left\langle I_{1}(t)\right\rangle+\left\langle I_{2}(t)\right\rangle\right] \\
& -\frac{\beta_{1}+\beta_{2}}{d} \Theta(t)+\sum_{i=1}^{2} \frac{M_{i}}{t}
\end{aligned}
$$

where $M_{i}(t)=\int_{0}^{t} \sigma_{i} S(\tau) \mathrm{d} B_{i}(\tau)$.

The inequality (21) can be rewritten as

$$
\begin{aligned}
\left\langle I_{1}(t)\right\rangle+\left\langle I_{2}(t)\right\rangle \geq & \frac{1}{\Delta_{\max }}\left[\sum _ { i = 1 } ^ { 2 } ( 1 + a _ { i } \frac { A } { d } ) ( d + \alpha _ { i } + r _ { i } ) \left(\frac{\beta_{i} A}{\left(d+a_{i} A\right)\left(d+\alpha_{i}+r_{i}\right)}\right.\right. \\
& \left.-\frac{\sigma_{i}^{2} A^{2}}{2\left(d+a_{i} A\right)^{2}\left(d+\alpha_{i}+r_{i}\right)}-1\right)-\frac{V(t)}{t}+\frac{V(0)}{t} \\
& \left.-\frac{\beta_{1}+\beta_{2}}{d} \Theta(t)+\sum_{i=1}^{2} \frac{M_{i}}{t}\right]
\end{aligned}
$$




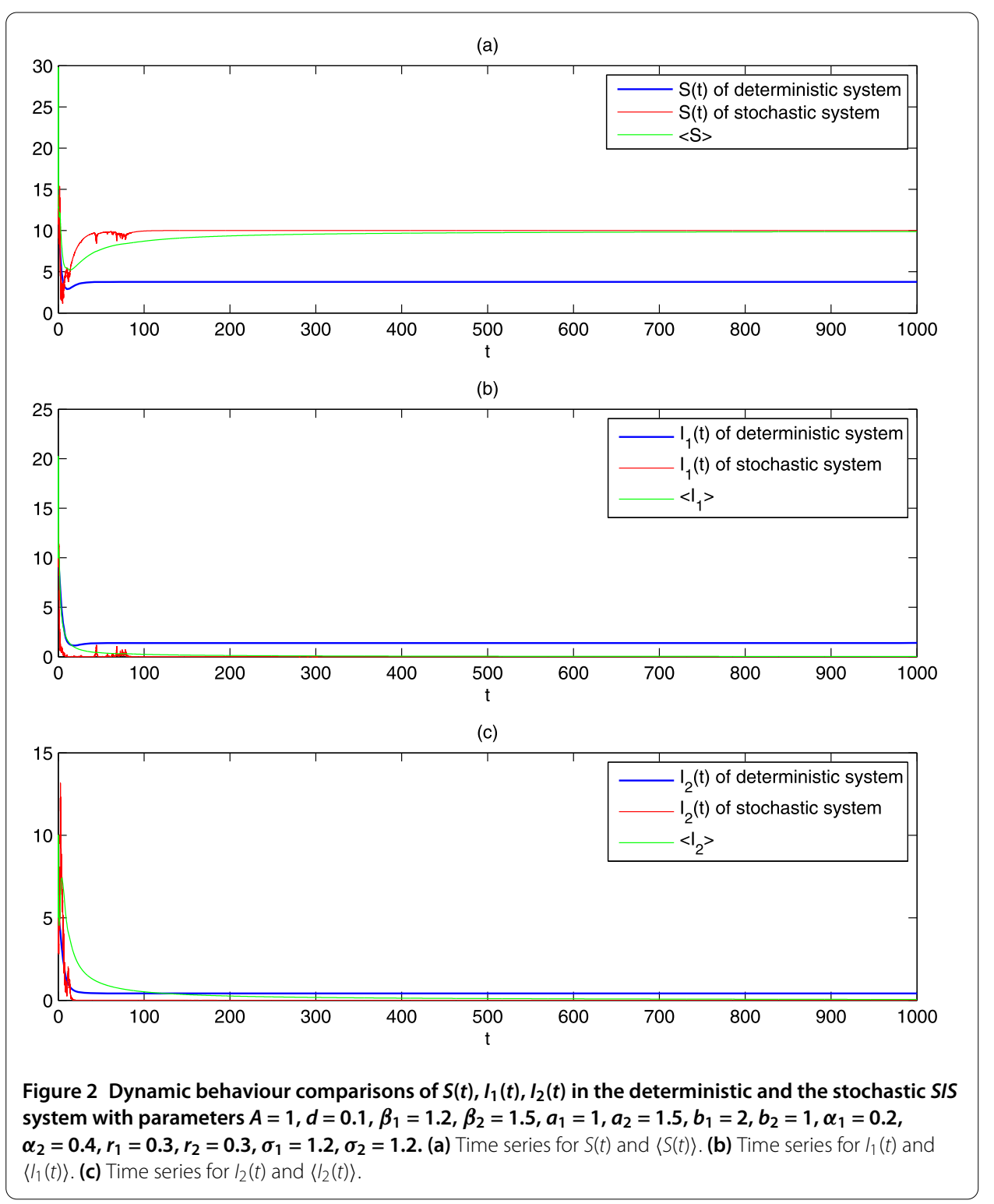

By Lemma 2.3, we have $\lim _{t \rightarrow+\infty} \frac{M_{i}(t)}{t}=0$, for $i=1,2$. According to Lemma 2.2, one can see that $\lim _{t \rightarrow+\infty} \Theta(t)=0$ and $\lim _{t \rightarrow+\infty} \frac{V(t)}{t}=0$.

Taking the inferior limit of both sides of (22) yields

$$
\liminf _{t \rightarrow+\infty}\left\langle I_{1}(t)+I_{2}(t)\right\rangle \geq \frac{1}{\Delta_{\max }} \sum_{i=1}^{2}\left(1+a_{i} \frac{A}{d}\right)\left(d+\alpha_{i}+r_{i}\right)\left(R_{i}^{*}-1\right)>0 .
$$

This completes the proof of Theorem 4.3.

Remark 4.3 Theorem 4.3 shows that both diseases will prevail if the white noise disturbances are small enough such that $\mathcal{R}_{i}^{*}>1$, conversely, if the white noise disturbances are large enough, then both diseases will become extinct. This implies that the stochastic disturbance may cause epidemic diseases to die out. 


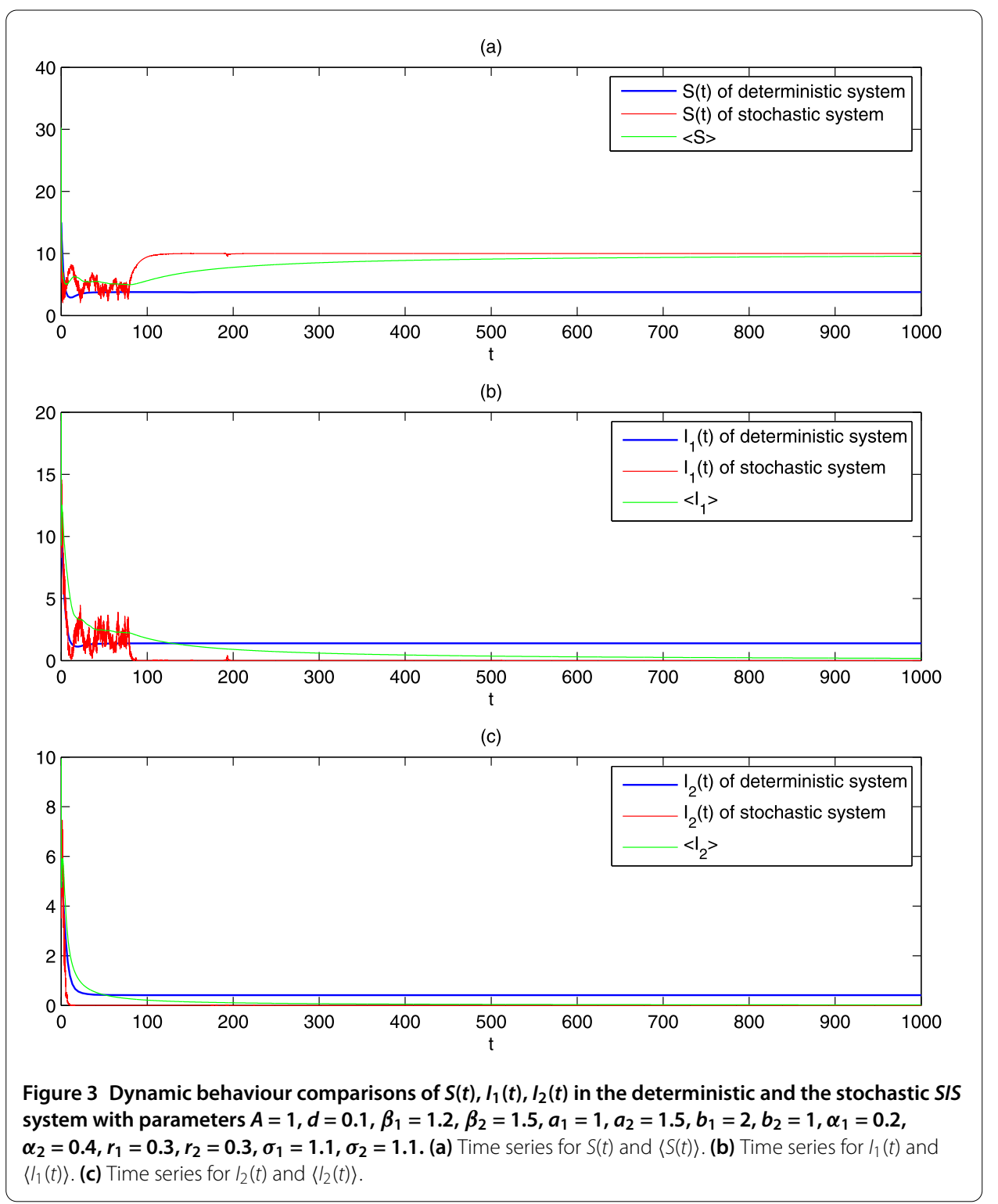

\section{Conclusion and simulations}

This paper proposed two SIS epidemic models with Beddington-DeAngelis incidence rate and double epidemic hypothesis from the point of view of deterministic and stochastic aspect. The threshold dynamics of both two systems were investigated and the conditions for extinction and permanence of both epidemic diseases were obtained. From Theorems 4.1 and 4.2, it can be seen that there is a significant difference between the thresholds of the stochastic system and the deterministic system, from which it can be concluded that the conditions for two epidemic diseases to go to extinction in the stochastic system are weaker than those of the deterministic system.

To illustrate the dynamic difference between the deterministic system and the stochastic system, we next carry out some numerical simulations of these cases with respect to different noise disturbance intensity using the Euler Maruyama (EM) method [36, 79]. 


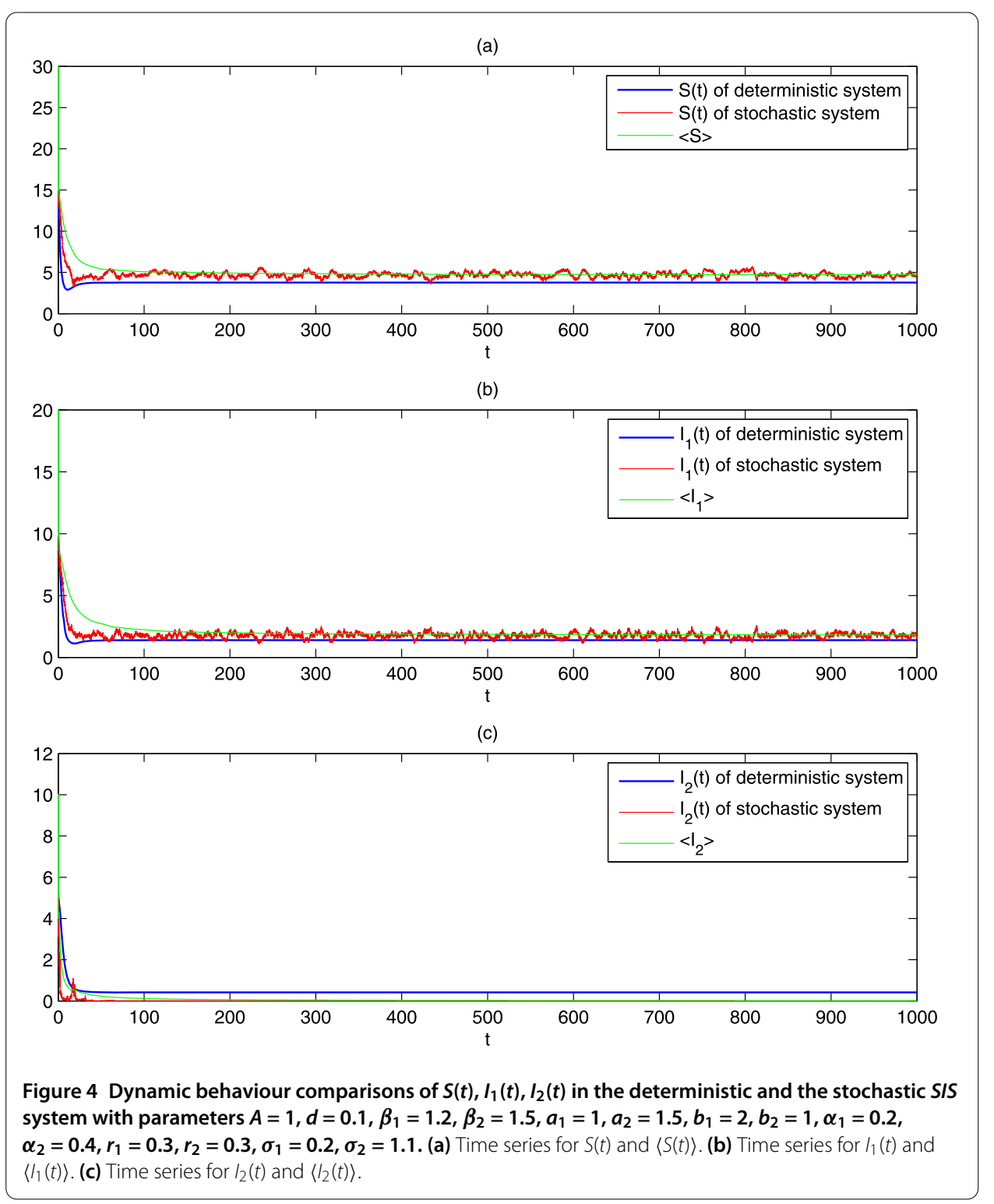

Choose the parameters in system (3) and system (4) as follows:

$$
\begin{array}{ll}
A=1, & d=0.1, \quad \beta_{1}=1.2, \\
\beta_{2}=1.5, & a_{1}=1, \quad a_{2}=1.5, \\
b_{1}=2, & b_{2}=1, \quad \alpha_{1}=0.2, \\
\alpha_{2}=0.4, & r_{1}=r_{2}=0.9 .
\end{array}
$$

A simple computation shows that $R_{1}=0.9091, R_{2}=0.6696$, then system (3) has a stable infection-free equilibrium $E_{0}(10,0,0)$, which implies that the two diseases of system (3) will die out ultimately (see Figure 1(a)). If we change $r_{1}=0.9$ to $r_{1}=0.3$, in this case, by simple calculation it can be found that $R_{1}=1.8182, R_{2}=0.6696$, the infection-free equilibrium $E_{1}(4.6,1.8,0)$ of system (3) is stable, which implies that the disease $I_{2}$ of system 


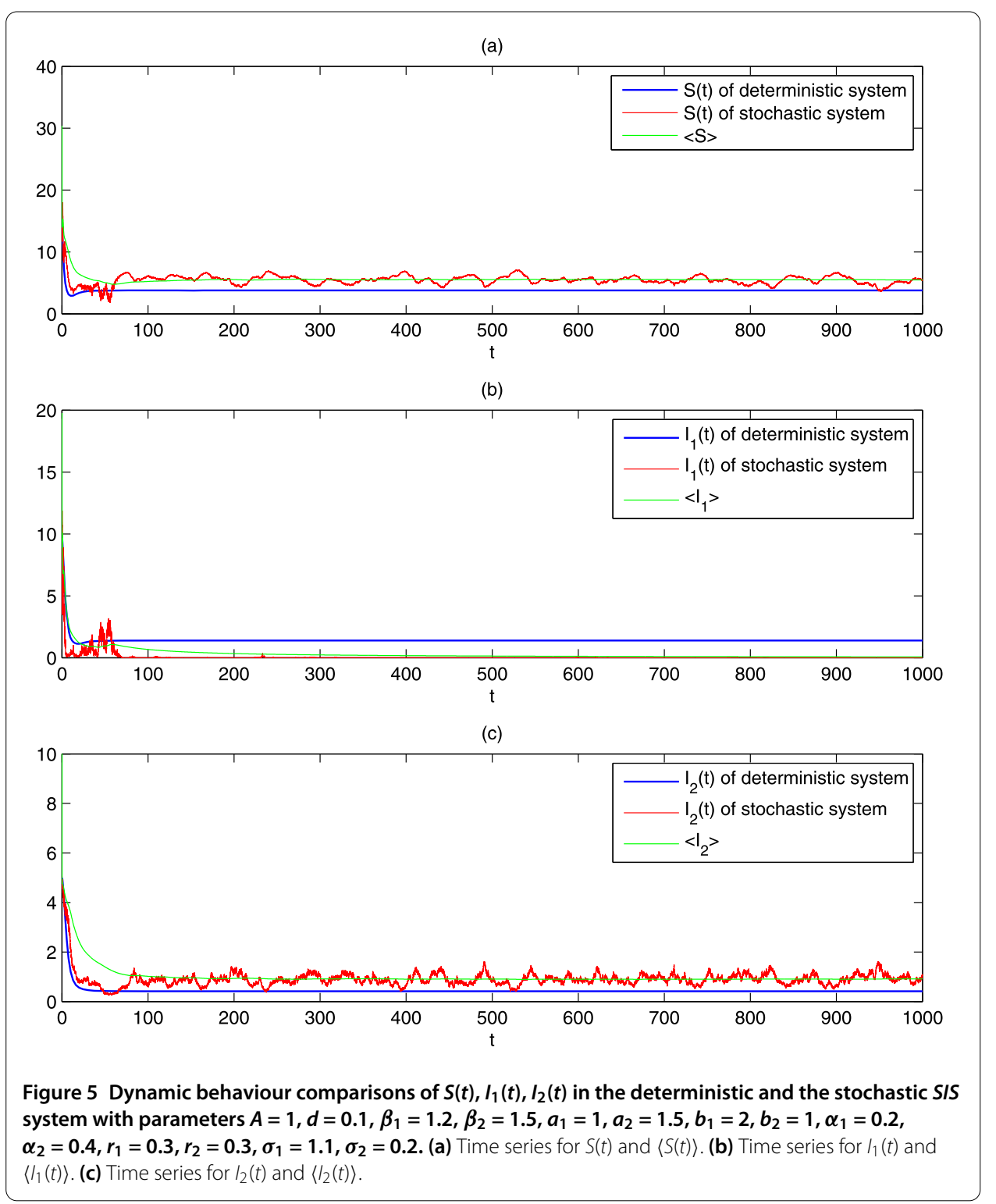

(3) will die out ultimately and the disease $I_{1}$ of system (3) will be persistent ultimately (see Figure 1(b)). If we update $r_{2}=0.9$ to $r_{2}=0.3$, in this case, $R_{1}=0.9091, R_{2}=1.1719$, the infection-free equilibrium $E_{1}(5.2174,0,0.9565)$ of system (3) is stable, which implies the disease $I_{1}$ of system (3) will die out ultimately and the disease $I_{2}$ of system (3) will be persistent ultimately (see Figure 1(c)). If we change $r_{1}=0.9, r_{2}=0.9$ to $r_{1}=0.3, r_{2}=0.3$, respectively, in this case, $R_{1}=1.8182, R_{2}=1.1719$, then (3) has a stable infection equilibrium $E^{*}(3.7714,1.3857,0.4143)$, which implies that two diseases of model (3) will be persistent ultimately (see Figure 1(d)).

Next, we consider the effect of stochastic white noise based on the persistent system. Let us choose both $\sigma_{1}$ and $\sigma_{2}$ as 1.2, in this case, $\sigma_{1}$ and $\sigma_{2}$ satisfy $\sigma_{i}>\frac{\beta_{i}}{\sqrt{2\left(d+\alpha_{i}+r_{i}\right)}}, i=1,2$. By Theorem 4.1, two diseases of system (4) will die out ultimately under a large white noise disturbance (see Figure 2(b) and Figure 2(c)). If we reduce both intensities of noise $\sigma_{1}, \sigma_{2}$ to 1.1 , in this case, by simple calculation it can be found that $R_{1}^{*}=0.9848, R_{2}^{*}=0.8765$, 


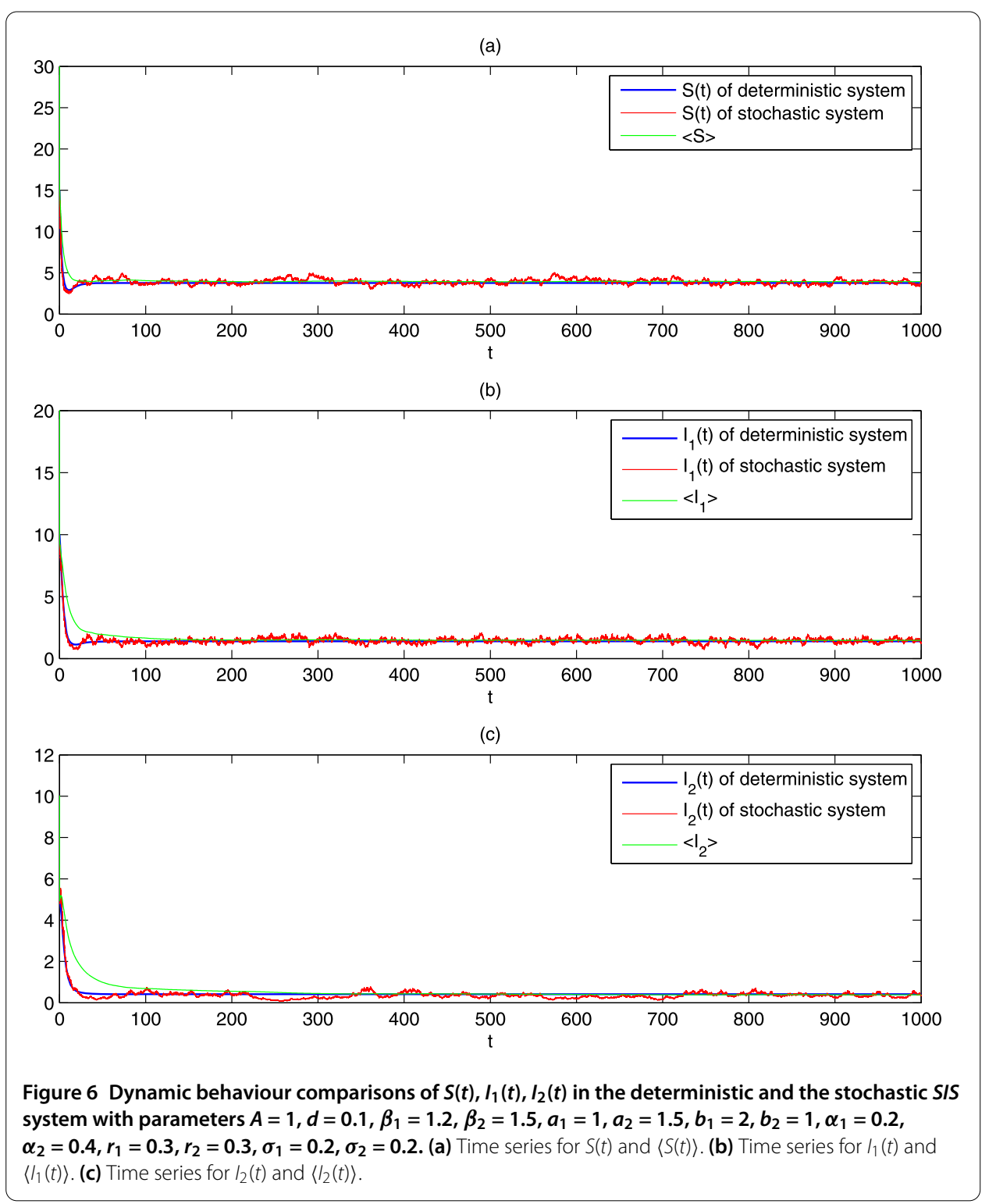

$\sigma_{1}$ and $\sigma_{2}$ satisfy $\sigma_{i} \leq \sqrt{\frac{\beta_{i}\left(A a_{i}+d\right)}{A}}$, where $i=1,2$. Then from Theorem 4.2, two diseases of system (4) will die out ultimately (see Figure 3(b) and Figure 3(c)).

If we reduce the intensity of noise $\sigma_{1}$ to 0.2 and keep the other system parameters the same as that in Figure 3, by computation, we have $R_{1}^{*}=1.7906, R_{2}^{*}=0.8765$, and $\sigma_{2}$ satisfy $\sigma_{2} \leq \sqrt{\frac{\beta_{2}\left(A a_{2}+d\right)}{A}}$. Then from Theorem 4.3, the disease $I_{2}(t)$ of system (4) will die out ultimately (see Figure $4(\mathrm{c})$ ) and the disease $I_{1}(t)$ of system (3) will be persistent ultimately (see Figure 4(b)). On the contrary, if we keep the intensity of noise of $\sigma_{1}=1.1$ and reduce the intensity of noise $\sigma_{2}$ to 0.2 , we can conclude that the disease $I_{1}(t)$ of system (4) will die out ultimately (see Figure 5(b)) and the disease $I_{2}(t)$ of system (3) will be persistent ultimately (see Figure 5(c)). Finally, if we respectively reduce the intensities of noise $\sigma_{1}$ and $\sigma_{2}$ at the same time, from 1.1 to 0.2 , by computation, we have $R_{1}^{*}=1.7906, R_{2}^{*}=1.1612$, then from Theorem 4.3, the two diseases of system (4) will be persistent ultimately (see Figure 6(b) and Figure 6(c)). 


\section{Acknowledgements}

This work is supported by Shandong Provincial Natural Science Foundation (No. ZR2015AQ001), National Natural Science Foundation of China (No. 11371230), Project for Higher Educational Science and Technology Program of Shandong Province (No. J13LI05), Research Funds for Joint Innovative Center for Safe and Effective Mining Technology and Equipment of Coal Resources by Shandong Province and SDUST Research Fund (2014TDJH102).

\section{Competing interests}

The authors declare that they have no competing interests.

Authors' contributions

All authors worked together to produce the results and read and approved the final manuscript.

\section{Author details}

'College of Mathematics and Systems Science, Shandong University of Science and Technology, Qianwangang Road 579, Qingdao, 266590, P.R. China. ${ }^{2}$ State Key Laboratory of Mining Disaster Prevention and Control Co-founded by Shandong Province and the Ministry of Science and Technology, Shandong University of Science and Technology, Qianwangang Road 579, Qingdao, 266590, P.R. China. ${ }^{3}$ School of Mathematics and Physics, University of Science and Technology Beijing, 30 Xue Yuan Road, Beijing, 100083, P.R. China. ${ }^{4}$ Department of Mathematics, University of Ruhuna, Matara, 81000, Sri Lanka.

\section{Publisher's Note}

Springer Nature remains neutral with regard to jurisdictional claims in published maps and institutional affiliations.

Received: 16 April 2017 Accepted: 19 July 2017 Published online: 04 August 2017

\section{References}

1. Hamer, WH: Epidemic disease in England. Lancet 1, 733-739 (1906)

2. Brauer, F, Castillo-Chavez, C: Mathematical Models in Population Biology and Epidemiology. Texts in Applied Mathematics. Springer, New York (2001)

3. Bernoulli, D, Blower, S: An attempt at a new analysis of the mortality caused by smallpox and of the advantages of inoculation to prevent it. Rev. Med. Virol. 14(5), 275-288 (2004)

4. Ross, RA: The Prevention of Malaria. Murray, London (1911)

5. May, RM, Anderson, RM, McLean, AR: Possible demographic consequences of HIV/AIDS epidemics. I. Assuming HIV infection always leads to AIDS. Math. Biosci. 90(1)، 475-505 (1988)

6. Anderson, RM, May, RM: Infectious Diseases of Humans: Dynamics and Control. Oxford University Press, Oxford (1992)

7. Zhang, T, Ma, W, Meng, X, Zhang, T: Periodic solution of a prey-predator model with nonlinear state feedback control. Appl. Math. Comput. 266, 95-107 (2015)

8. Meng, X, Zhang, L: Evolutionary dynamics in a Lotka-Volterra competition model with impulsive periodic disturbance. Math. Methods Appl. Sci. 39(2), 177-188 (2016)

9. Cheng, H, Zhang, T: A new predator-prey model with a profitless delay of digestion and impulsive perturbation on the prey. Appl. Math. Comput. 217(22), 9198-9208 (2011)

10. Cheng, H, Zhang, T, Wang, F: Existence and attractiveness of order one periodic solution of a Holling I predator-prey model. Abstr. Appl. Anal. 2012, Article ID 126018 (2012)

11. Zhang, T, Ma, W, Meng, X: Global dynamics of a delayed chemostat model with harvest by impulsive flocculant input. Adv. Differ. Equ. 2017(1), 115 (2017)

12. $\mathrm{Xu}, \mathrm{X}$ : A deformed reduced semi-discrete Kaup-Newell equation, the related integrable family and Darboux transformation. Appl. Math. Comput. 251, 275-283 (2015)

13. Dong, H, Zhang, Y, Zhang, X: The new integrable symplectic map and the symmetry of integrable nonlinear lattice equation. Commun. Nonlinear Sci. Numer. Simul. 36, 354-365 (2016)

14. Zhang, Y, Dong, H, Zhang, X, Yang, H: Rational solutions and lump solutions to the generalized-dimensional shallow water-like equation. Comput. Math. Appl. 73(2), 246-252 (2017)

15. Dong, H, Guo, B, Yin, B: Generalized fractional supertrace identity for Hamiltonian structure of NLS-MKdV hierarchy with self-consistent sources. Anal. Math. Phys. 6(2), 199-209 (2016)

16. Fang, Y, Dong, $\mathrm{H}, \mathrm{Hou}, \mathrm{Y}, \mathrm{Kong}, \mathrm{Y}$ : Frobenius integrable decompositions of nonlinear evolution equations with modified term. Appl. Math. Comput. 226, 435-440 (2014)

17. Meng, X, Chen, L, Wu, B: A delay sir epidemic model with pulse vaccination and incubation times. Nonlinear Anal., Real World Appl. 11(1), 88-98 (2010)

18. Zhang, T, Meng, X, Zhang, T: Global analysis for a delayed SIV model with direct and environmental transmissions. J. Appl. Anal. Comput. 6(2), 1479-1491 (2016)

19. Cui, Y: Uniqueness of solution for boundary value problems for fractional differential equations. Appl. Math. Lett. 51 48-54 (2016)

20. Bai, Z, Dong, X, Yin, C: Existence results for impulsive nonlinear fractional differential equation with mixed boundary conditions. Bound. Value Probl. 2016(1), 63 (2016)

21. Cui, Y, Zou, Y: An existence and uniqueness theorem for a second order nonlinear system with coupled integral boundary value conditions. Appl. Math. Comput. 256, 438-444 (2015)

22. Bai, Z, Dong, X, Yin, C: Monotone iterative method for fractional differential equations. Electron. J. Differ. Equ. 2016, 6 (2016)

23. Zou, Y, Cui, Y: Existence results for a functional boundary value problem of fractional differential equations. Adv. Differ. Equ. 2013(1), 233 (2013)

24. Zhang, T, Zhang, T, Meng, X: Stability analysis of a chemostat model with maintenance energy. Appl. Math. Lett. 68 $1-7(2017)$ 
25. Kermack, WO, McKendrick, AG: Contributions to the mathematical theory of epidemics - I. Bull. Math. Biol. 53(1), 33-55 (1991)

26. Kermack, WO, McKendrick, AG: Contributions to the mathematical theory of epidemics: II. Further studies of the problem of endemicity. Bull. Math. Biol. 53(1), 89-118 (1991)

27. Xu, R, Ma, Z: Global stability of a SIR epidemic model with nonlinear incidence rate and time delay. Nonlinear Anal., Real World Appl. 10(5), 3175-3189 (2009)

28. Xu, R, Zhang, S, Zhang, F: Global dynamics of a delayed SElS infectious disease model with logistic growth and saturation incidence. Math. Methods Appl. Sci. 39, 3294-3308 (2016)

29. Zhang, T, Teng, Z: Global asymptotic stability of a delayed SEIRS epidemic model with saturation incidence. Chaos Solitons Fractals 37(5), 1456-1468 (2008)

30. Liu, W, Levin, SA, Iwasa, Y: Influence of nonlinear incidence rates upon the behavior of SIRS epidemiological models. J. Math. Biol. 23(2), 187-204 (1986)

31. Liu, W, Hethcote, HW, Levin, SA: Dynamical behavior of epidemiological models with nonlinear incidence rates. J. Math. Biol. 25(4), 359-380 (1987)

32. Hethcote, HW, Lewis, MA, van den Driessche, P: An epidemiological model with a delay and a nonlinear incidence rate. J. Math. Biol. 27(1), 49-64 (1989)

33. Ruan, S, Wang, W: Dynamical behavior of an epidemic model with a nonlinear incidence rate. J. Differ. Equ. 188(1), 135-163 (2003)

34. Chen, L, Hu, Z, Liao, F: The stability of an SEIR model with nonlinear Beddington-DeAngelis incidence, vertical transmission and time delay. J. Aanhui Norm. Univ. 39(1), 26-32 (2016)

35. Wang, L, Li, J-Q: Global stability of an epidemic model with nonlinear incidence rate and differential infectivity. Appl. Math. Comput. 161(3), 769-778 (2005)

36. Mao, X: Stochastic Differential Equations and Applications. Horwood, Chichester (2008)

37. Artalejo, JR, Economou, A, Lopez-Herrero, MJ: On the number of recovered individuals in the SIS and SIR stochastic epidemic models. Math. Biosci. 228(1), 45-55 (2010)

38. Bacaër, N: On the stochastic SIS epidemic model in a periodic environment. J. Math. Biol. 71(2), $491-511$ (2014)

39. Meng, X: Stability of a novel stochastic epidemic model with double epidemic hypothesis. Appl. Math. Comput. 217(2), 506-515 (2010)

40. Beretta, E, Kolmanovskii, V, Shaikhet, L: Stability of epidemic model with time delays influenced by stochastic perturbations. Math. Comput. Simul. 45(3-4), 269-277 (1998)

41. Yu, J, Jiang, D, Shi, N: Global stability of two-group SIR model with random perturbation. J. Math. Anal. Appl. 360(1), 235-244 (2009)

42. Ji, C, Jiang, D: Threshold behaviour of a stochastic SIR model. Appl. Math. Model. 38(21-22), 5067-5079 (2014)

43. Feng, T, Meng, X, Liu, L, Gao, S: Application of inequalities technique to dynamics analysis of a stochastic eco-epidemiology model. J. Inequal. Appl. 2016(1), 327 (2016)

44. Ma, H, Jia, Y: Stability analysis for stochastic differential equations with infinite Markovian switchings. J. Math. Anal. Appl. 435(1), 593-605 (2016)

45. Zhao, W, Li, J, Zhang, T, Meng, X, Zhang, T: Persistence and ergodicity of plant disease model with Markov conversion and impulsive toxicant input. Commun. Nonlinear Sci. Numer. Simul. 48, 70-84 (2017)

46. Tuckwell, HC, Williams, RJ: Some properties of a simple stochastic epidemic model of SIR type. Math. Biosci. 208(1), 76-97 (2007)

47. Cai, Y, Kang, Y, Banerjee, M, Wang, W: A stochastic SIRS epidemic model with infectious force under intervention strategies. J. Differ. Equ. 259(12), 7463-7502 (2015)

48. Gray, A, Greenhalgh, D, Mao, X, Pan, J: The SIS epidemic model with Markovian switching. J. Math. Anal. Appl. 394(2), 496-516 (2012)

49. Zhang, X, Jiang, D, Alsaedi, A, Hayat, T: Stationary distribution of stochastic SIS epidemic model with vaccination under regime switching. Appl. Math. Lett. 59, 87-93 (2016)

50. Meng, X, Zhao, S, Feng, T, Zhang, T: Dynamics of a novel nonlinear stochastic SIS epidemic model with double epidemic hypothesis. J. Math. Anal. Appl. 433(1), 227-242 (2016)

51. Tornatore, E, Buccellato, SM, Vetro, P: Stability of a stochastic sir system. Phys. A, Stat. Mech. Appl. 354, 111-126 (2005)

52. Chang, Z, Meng, $X, L u, X$ : Analysis of a novel stochastic SIRS epidemic model with two different saturated incidence rates. Phys. A, Stat. Mech. Appl. 472, 103-116 (2017)

53. Gray, A, Greenhalgh, D, Hu, L, Mao, X, Pan, J: A stochastic differential equation SIS epidemic model. SIAM J. Appl. Math 71(3), 876-902 (2011)

54. Lin, Y, Jiang, D: Long-time behaviour of a perturbed SIR model by white noise. Discrete Contin. Dyn. Syst., Ser. B 18(7), 1873-1887 (2013)

55. Schurz, H, Tosun, K: Stochastic asymptotic stability of SIR model with variable diffusion rates. J. Dyn. Differ. Equ. 27(1), 69-82 (2015)

56. Lu, Q: Stability of SIRS system with random perturbations. Phys. A, Stat. Mech. Appl. 388(18), 3677-3686 (2009)

57. Wei, F, Liu, J: Long-time behavior of a stochastic epidemic model with varying population size. Phys. A, Stat. Mech. Appl. 470, 146-153 (2017)

58. Dalal, N, Greenhalgh, D, Mao, X: A stochastic model of AIDS and condom use. J. Math. Anal. Appl. 325(1), 36-53 (2007)

59. Xu, C: Global threshold dynamics of a stochastic differential equation SIS model. J. Math. Anal. Appl. 447(2), 736-757 (2017)

60. Lahrouz, A, Settati, A: Necessary and sufficient condition for extinction and persistence of SIRS system with random perturbation. Appl. Math. Comput. 233, 10-19 (2014)

61. Lahrouz, A, Settati, A: Qualitative study of a nonlinear stochastic SIRS epidemic system. Stoch. Anal. Appl. 32(6), 992-1008 (2014)

62. Zhao, D, Zhang, T, Yuan, S: The threshold of a stochastic SIVS epidemic model with nonlinear saturated incidence. Phys. A, Stat. Mech. Appl. 443, 372-379 (2016)

63. Zhao, Y, Lin, Y, Jiang, D, Mao, X, Li, Y: Stationary distribution of stochastic SIRS epidemic model with standard incidence. Discrete Contin. Dyn. Syst., Ser. B 21(7), 2363-2378 (2016) 
64. Miao, A, Zhang, J, Zhang, T, Sampath Aruna Pradeep, BG: Threshold dynamics of a stochastic SIR model with vertical transmission and vaccination. Comput. Math. Methods Med. 2017, Article ID 4820183 (2017)

65. Lahrouz, A, Settati, A, Akharif, A: Effects of stochastic perturbation on the SIS epidemic system. J. Math. Biol. 74(1), 469-498 (2017)

66. Liu, Q, Jiang, D, Shi, N, Hayat, T, Alsaedi, A: Stationary distribution and extinction of a stochastic SIRS epidemic model with standard incidence. Phys. A, Stat. Mech. Appl. 469, 510-517 (2017)

67. Dieu, NT, Nguyen, DH, Du, NH, Yin, G: Classification of asymptotic behavior in a stochastic SIR model. SIAM J. Appl. Dyn. Syst. 15(2), 1062-1084 (2016)

68. Zhao, Y, Jiang, D: The threshold of a stochastic SIS epidemic model with vaccination. Appl. Math. Comput. 243, 718-727 (2014)

69. Jiang, D, Liu, Q, Shi, N, Hayat, T, Alsaedi, A, Xia, P: Dynamics of a stochastic HIV-1 infection model with logistic growth. Phys. A, Stat. Mech. Appl. 469, 706-717 (2017)

70. Cai, Y, Kang, Y, Banerjee, M, Wang, W: A stochastic epidemic model incorporating media coverage. Commun. Math. Sci. 14(4), 893-910 (2016)

71. Du, NH, Nhu, NN: Permanence and extinction of certain stochastic SIR models perturbed by a complex type of noises. Appl. Math. Lett. 64, 223-230 (2017)

72. Liu, Q, Chen, Q: Analysis of the deterministic and stochastic SIRS epidemic models with nonlinear incidence. Phys. A Stat. Mech. Appl. 428, 140-153 (2015)

73. Zhang, X, Jiang, D, Hayat, T, Ahmad, B: Dynamics of a stochastic SIS model with double epidemic diseases driven by Lévy jumps. Phys. A, Stat. Mech. Appl. 471, 767-777 (2017)

74. Li, C, Pei, Y, Liang, X, Fang, D: A stochastic toxoplasmosis spread model between cat and oocyst with jumps process. Commun. Math. Biol. Neurosci. 2016, 18 (2016)

75. Zhou, Y, Yuan, S, Zhao, D: Threshold behavior of a stochastic SIS model with jumps. Appl. Math. Comput. 275, $255-267(2016)$

76. Jiang, D, Ji, C, Shi, N, Yu, J: The long time behavior of DI SIR epidemic model with stochastic perturbation. J. Math Anal. Appl. 372(1), 162-180 (2010)

77. Liu, M, Bai, C, Wang, K: Asymptotic stability of a two-group stochastic SEIR model with infinite delays. Commun. Nonlinear Sci. Numer. Simul., 19(10), 3444-3453 (2014)

78. Liu, Q, Jiang, D, Shi, N, Hayat, T, Alsaedi, A: Asymptotic behavior of a stochastic delayed SEIR epidemic model with nonlinear incidence. Phys. A, Stat. Mech. Appl. 462, 870-882 (2016)

79. Kloeden, PE, Platen, E: Higher-order implicit strong numerical schemes for stochastic differential equations. J. Stat. Phys. 66(1), 283-314 (1992)

\section{Submit your manuscript to a SpringerOpen ${ }^{\circ}$ journal and benefit from:}

- Convenient online submission

- Rigorous peer review

- Open access: articles freely available online

- High visibility within the field

- Retaining the copyright to your article

Submit your next manuscript at $\boldsymbol{\nabla}$ springeropen.com 